Measured Strains

$S_{X}=1.17$
$S_{Y}=0.97$
$S_{Z}=0.52$

maximum
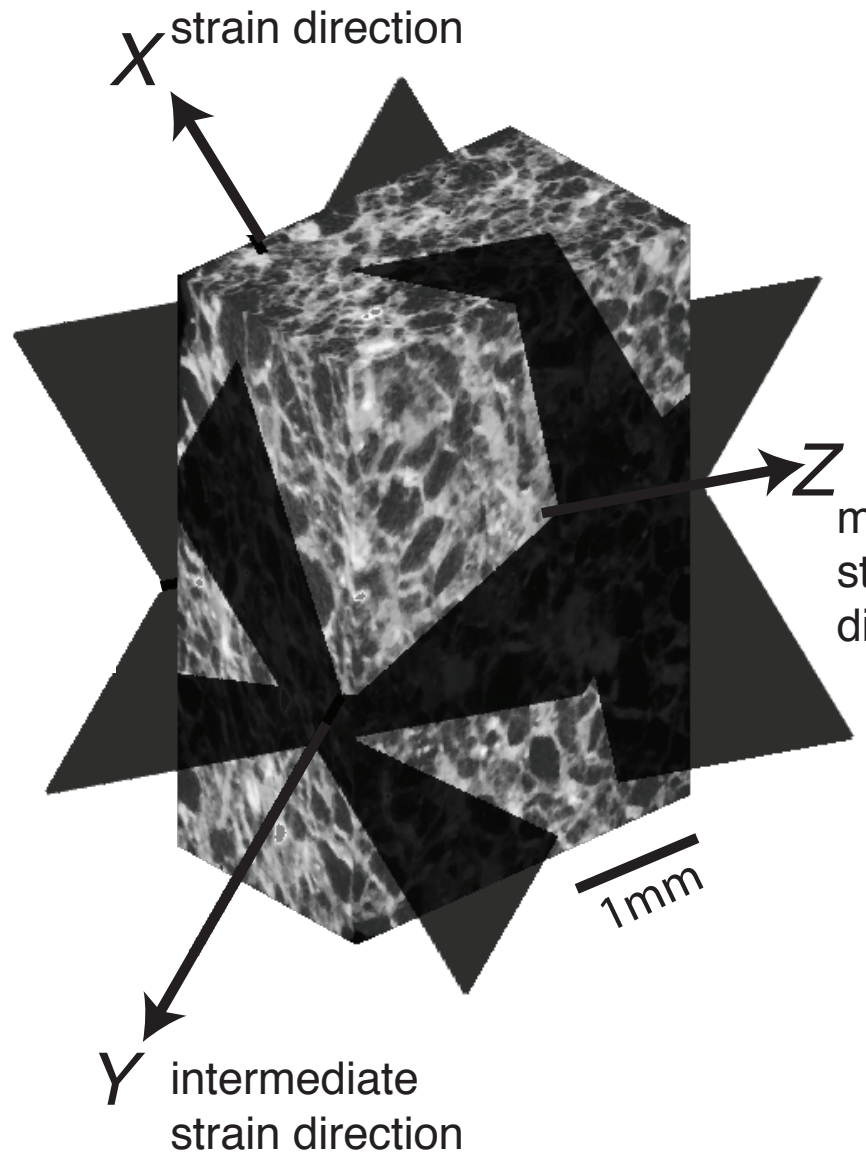

strain ellipsoid b) $X-Z$ Section

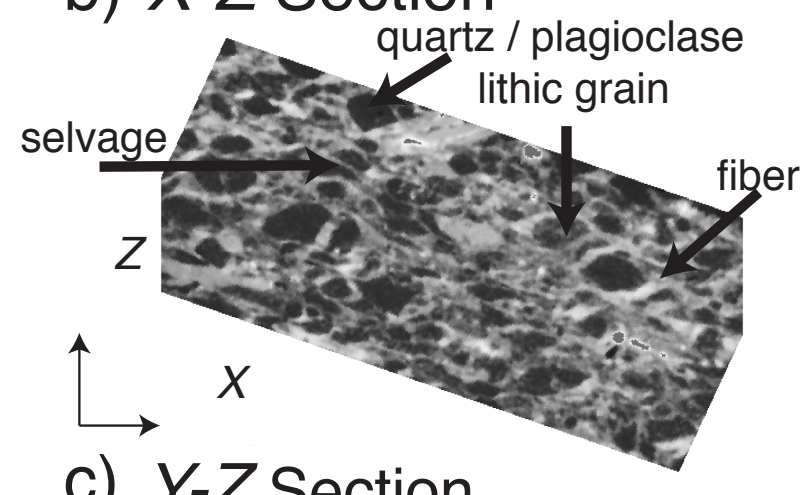

c) $Y$-Z Section

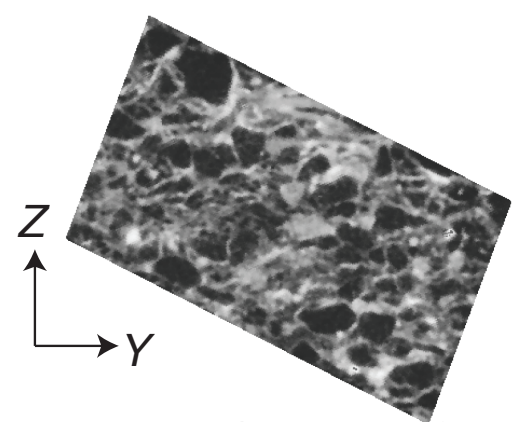

d) $X-Y$ Section

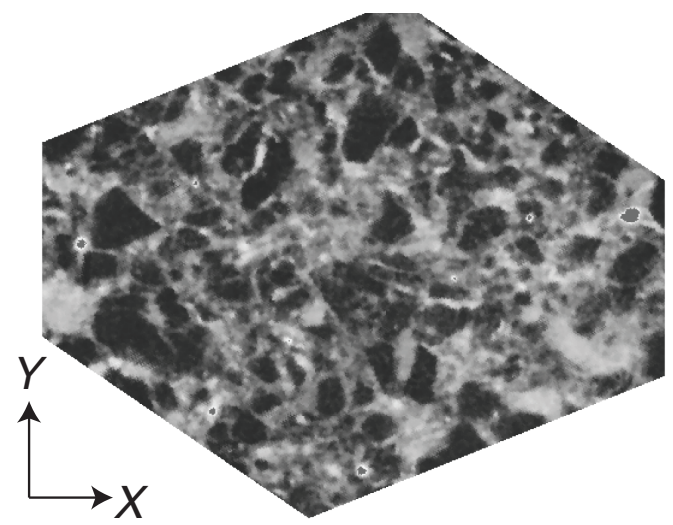




\section{An Autocorrelation Method for Three-Dimensional Strain Analysis}

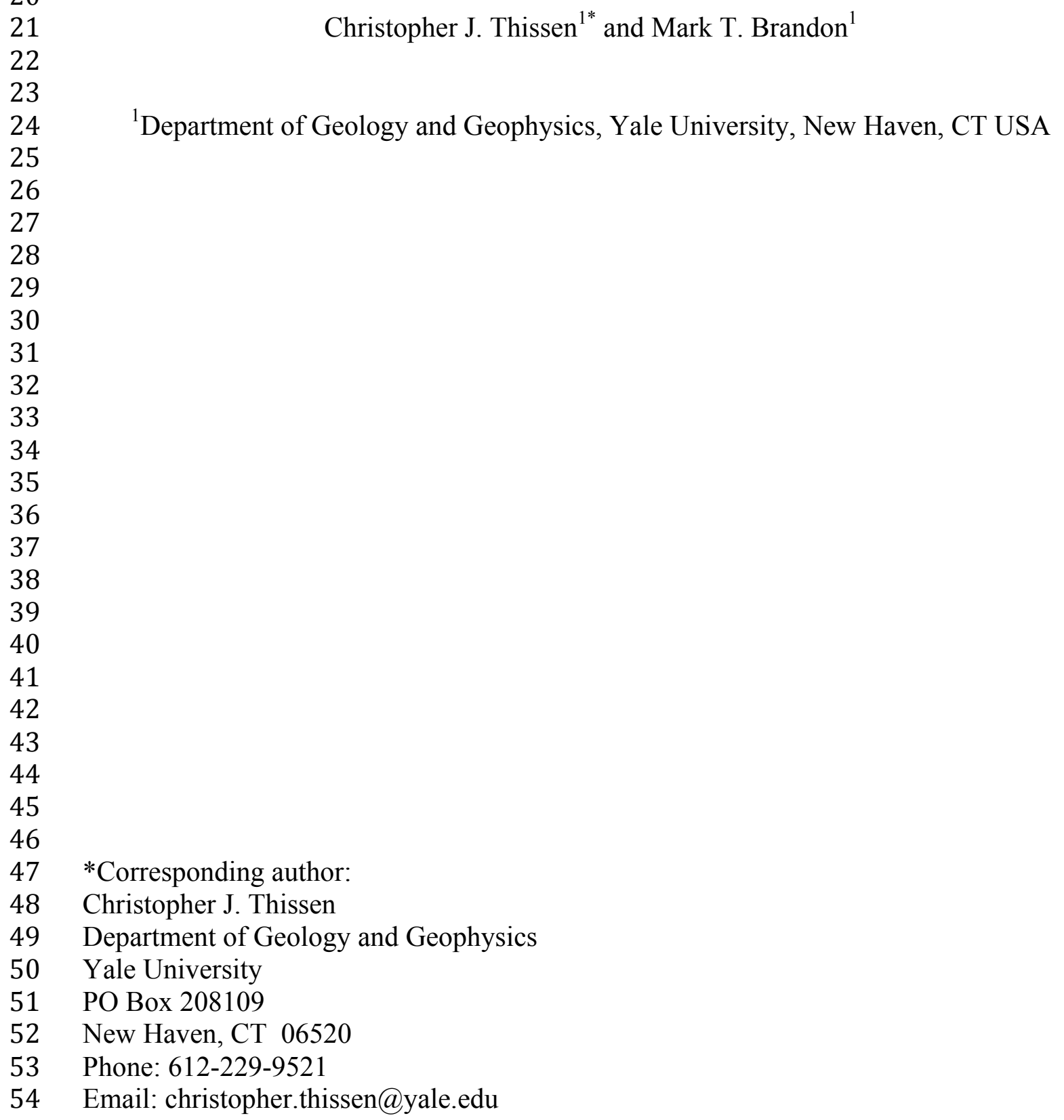

*Corresponding author:

${ }^{1}$ Department of Geology and Geophysics, Yale University, New Haven, CT USA

54 Email: christopher.thissen@yale.edu

56 keywords: X-ray tomography; finite strain; autocorrelation; pressure solution; 57 deformation; 


\section{Abstract}

59 We present a method of finite-strain estimation using the autocorrelation properties

60 of deformed rock. Autocorrelation is the correlation of an image with itself as a

61 function of an offset or lag. In two and three dimensions, the lag has both distance

62 and direction. Many geologic materials are initially isotropic, which means that the

63 autocorrelation function (ACF) will be initially isotropic as well. Deformation

64 imposes an anisotropic distortion, which can be measured using the Ramsay and

65 Fry strain methods for example, but can also be estimated from the ACF. X-ray

66 tomography now provides a rapid way to measure the three-dimensional ACF of a

67 geologic sample. Strain parameters are estimated by using a non-linear, best-fit

68 method to minimize the anisotropy in the ACF. The ACF method works best for

69 materials where the objects are internally uniform and contrast strongly with the

70 matrix and other grains. 
71 "So fine was the morning except for a streak of wind here and there that the sea and

72 sky looked all one fabric...." - Virginia Woolf, To the Lighthouse

73

74 1. Introduction

75 Geologists use the term fabric to refer to the spatial organization of features in a

76 rock (e.g., Heilbronner and Barrett, 2013). Rocks will commonly contain primary

77 fabrics, created at the time that the rock formed (e.g., bedding, cross-bedding,

78 cumulate layering, etc.), and secondary fabrics, formed by metamorphism and

79 deformation (e.g., metamorphic and melt segregation, foliation, lineation,

80 crenulation, folds, boudins, shear bands, faults, etc.). The autocorrelation function

81 (ACF) provides a generalized method for measuring the spatial dimensions and

82 anisotropy of almost all rock fabrics (e.g., Panozzo Heilbronner, 1992; Heilbronner

83 and Barrett, 2013).

84

85 Many methods of deformation analysis depend on statistical estimation of rock

86 fabrics (e.g., Ramsay and Huber, 1983). In fact, if rocks lacked spatially organized

87 fabrics, there would be no structure to analyze. The atmosphere on a sunny day is

88 one example of a material that exhibits a lack of contrast. The atmosphere flows as a

89 fluid, but on a clear day, it lacks any "memory" of its deformational history because

90 the air lacks any visible internal structure. Similarly, a material where each point is

91 independent of all other points, such as an image of white noise, also has no

92 information about its deformational history (Fry, 1979). An atmosphere that

93 contains both cloudy and clear air can preserve some information about its flow 
94 history. The reason is that the mixture of cloudy and clear air creates an internally

95 correlated structure, in that particles within a cloud are likely to be found near other

96 cloud particles, and clear air particles are likely to be found near other clear air

97 particles. The ACF provides a generalized method to quantify both the magnitude

98 and orientation of this correlation.

100 Our objective here is to relate ACF measurements to strain. Thus, it is important to

101 note that the ACF method, like many strain methods, requires well-understood

102 features that are statistically isotropic in the initial state and respond passively to

103 deformation. As with many strain methods, the success of the measurements

104 depends on finding samples that are consistent with these requirements.

106 Panozzo Heilbronner (1992) was first to propose using the ACF for measuring finite

107 strain. The approach has become more appealing given the availability of X-ray

108 tomography (e.g., Fusseis et al., 2014), which directly images the three-dimensional

109 fabric of a rock. Note that we use the term "image" in a generic sense to mean any

110 two-dimensional (2D) or three-dimensional (3D) field. In X-ray tomography, the

111 features in the rock are represented by the spatial variation in X-ray attenuation. A

112 3D attenuation field provides an ideal image for estimating autocorrelation in rock 113 samples.

115 For granular materials, deformation creates a directional variation in grain

116 diameter. The ACF reveals this fabric as a directional variation in the ACF length 
117 scale. In the simplest case, the axial ratio of the contours of the ACF equals the axial

118 ratios of the grains (Lado and Torquato, 1990; Torquato and Lado, 1991; Panozzo

119 Heilbronner 1992). Panozzo Heilbronner (1992) estimated finite strain by

120 measuring the ellipticity of contours of the ACF. Others have considered how the

121 ACF varies spatially throughout the sample and also as a function of lag

122 (Heilbronner, 2010; Svitelman and Dinariev, 2013).

123

124 We build on this previous work in three ways. First, we introduce a generalized

125 best-fit method for estimating the anisotropy of the ACF determined from a 2D or

126 3D image. Second, we develop a method for estimating the uncertainties associated

127 with the estimated parameters. Third, we consider both theoretical and practical

128 aspects for implementing this method to measure strain. Our analysis of the ACF

129 depends, in part, on the type of data analyzed, and so we begin with an overview of 130 X-ray tomography.

\section{2. X-ray Tomography}

133 X-ray tomography provides a 3D image of the X-ray attenuation structure of an

134 object at a micron scale (Maire and Withers, 2014). The time required to image a

135 sample is beam-line dependent, but ranges from several hours to 10 minutes or

136 faster (Rivers et al., 1999). X-ray tomography requires limited sample preparation

137 (e.g., Lindquist and Venkatarangan, 2000; Fusseis et al., 2014). It is non-destructive,

138 which is ideal for rare or valuable samples (e.g., Rivers et al., 1999; Ketcham and

139 Carlson, 2001; Ebel and Rivers, 2007), and for samples where other complementary 
140 analyses are needed (e.g., figure 1 in Hu et al., 2014). The following papers provide

141 an introduction to the method: Denison et al. (1997); Denison and Carlson, (1997);

142 Ketcham and Carlson (2001); Sutton et al., (2002); Carlson (2006); Jerram and

143 Higgins (2007); Baker et al. (2012); Cnudde and Boon (2013); Fusseis et al. (2014);

144 Maire and Withers (2014). Sample preparation, experimental design, choosing an

145 appropriate photon source, and image reconstruction are reviewed by Reeder and

146 Lanzirotti (2006) and Fusseis et al., (2014).

148 X-ray attenuation is a scalar field variable, meaning it is a single quantity that varies

149 spatially throughout the sample volume. A tomographic image, or tomogram, is

150 represented by discrete attenuation estimates at nodes within a 3D grid. Each node

151 lies at the center of a volume element called a voxel, which is the 3D equivalent of a

152 pixel (Carlson, 2006). The attenuation coefficient reported in each voxel is a

153 weighted average for material within the voxel (Hubbell and Seltzer, 2004; Gualda

154 et al., 2010).

155

156 X-ray attenuation varies throughout a heterogeneous sample. The example shown in

157 Figure 1 is a $1392 \times 1392 \times 1$ voxel slice from the $1392 \times 1392 \times 1040$ voxel grid that

158 spans a full sample volume. Attenuation is represented by a grayscale intensity (1-

159 256), with white (256) representing the largest attenuation. Note that the region

160 surrounding the sample is black because air has minimal attenuation.

161

162 Figure 1 here. 
164 The attenuation coefficient, $\mu$, is defined by

$$
\mu=-\frac{1}{I} \frac{\mathrm{d} I}{\mathrm{~d} s}
$$

166 where $s$ is the path length of the X-ray beam, and $I$ is the X-ray intensity (Hubble and

167 Selzter 2004). X-ray tomography is able to easily resolve features with attenuation

168 coefficients that differ by more than a factor of two (Ebel and Rivers, 2007; Gualda

169 et al., 2010). The coefficient $\mu$ scales linearly with density and as a power law with

170 atomic number (e.g., Denison et al., 1997a). Several calculators are available for

171 estimating $\mu$ (Hubbell and Seltzer, 2004; Chantler et al., 2005; Berger et al., 2010;

172 MuCalc spreadsheet: http://www.ctlab.geo.utexas.edu/software/index.php).

173 Combining X-ray attenuation with other kinds of data can further improve image

174 resolution (Huddlestone-Holmes and Ketcham, 2005; Boone et al., 2011). X-ray

175 tomography requires that the total attenuation through the sample is no more than

$17680 \%$ (Gualda et al., 2010), so suitable rock samples are commonly on the order of

177 centimeters in size. A contrast in attenuation coefficients, rather than the absolute

178 value, is important for successfully imaging fabrics using X-ray tomography For

179 example, most minerals have a strong contrast with quartz except for feldspar (Fig.

180 2). The beam energy can be adjusted to improve the contrast for some minerals. The

181 large jumps in the attenuation value are absorption edges, and correspond to the

182 ejection of electrons from specific electron shells (Gualda et al., 2010).

183

184 Figure 2 here. 
186 Many software programs have been developed to analyze 3D tomography data for

187 geologic materials: Quant3D (Ryan and Ketcham, 2002; Ketcham and Ryan, 2004),

188 Blob3D (Ketcham 2005a; 2005b), Ctsta (Liu et al., 2009), WinDICOM (O'Connor et

189 al., 2009), Morpho+ (Brabant et al., 2009), Pore3D (Brun et al., 2010), PhaseQuant

190 (Elangovan et al., 2012), YaDiv (Friese et al., 2013). Fusseis et al. (2014) reviews

191 these programs in more detail. In particular, Ketcham (2005a; 2005b) provides a

192 way to isolate the shape of segmented phases and analyze them for strain estimates.

193 Some authors have highlighted difficulties in estimating parameters using

194 segmentation methods (e.g., Iaasonov et al., 2009; Baveye et al., 2010; Iassonov and

195 Tuller, 2010; Andrä et al., 2012). Our method avoids these problems by using a

196 statistical description of the entire sample, independent of actual grain boundaries.

\section{3. The Autocorrelation Function}

199 To provide a more intuitive understanding of the ACF, we begin by considering a

200 few qualitative examples. Fig. 3a shows an average of yearbook photos for high-

201 school women in the 1967 class in Fort Worth, Texas (Salavon, 1998). The

202 individuals are unrecognizable, but the average shows a clear structure that is

203 immediately identifiable as a generic portrait. The average also shows hints about

204 the dress and hairstyle typical for 1967.

205

206 Figure 3 here. 
208 Similarly, Fig. 3b shows an average of 200 tomogram slices, or approximately 392

$209 \mu \mathrm{m}$, for the sandstone sample in Fig. 1. If the image volume had no correlated

210 structures (e.g., no grains or foliation), then each pixel in the image would be

211 independent of all the other pixels, and the average image in Fig. $3 \mathrm{~b}$ would be a

212 statistically uniform shade of gray. However, the image shows areas with distinct

213 pixel intensities, which indicate spatial correlations over the 200 slices.

215 Figure 4 here.

217 Considering autocorrelation more formally, the ACF is represented by the notation,

$218 \rho(\mathbf{r})$, where the lag vector $\mathbf{r}$ indicates the offset between the image and its copy, and

219 the autocorrelation statistic $\rho$ is a measure of the overall similarity of the images in

220 this offset relationship. $\rho$ is limited to the range -1 to +1 . A value of +1 means that

221 the image and its offset copy match exactly, at a pixel or voxel level (i.e. perfect

222 correlation). A value of 0 means that the image and its offset copy have no statistical

223 similarity. A value of -1 means that the image and its offset copy are complete

224 opposites (i.e., perfect anticorrelation). The ACFs for most real images usually range

225 from 0 to +1 .

226

227 Several simple examples illustrate basic features of the ACF (Fig. 4). The examples

228 are composed of two phases, which appear as black circles surrounded by a white

229 background. It is helpful to envision the autocorrelation at a specific lag as the

230 overlapping area of the black circles in two identical, offset sheets (Panozzo- 
231 Heilbronner, 1992). However, when the ACF is calculated, the image is rescaled so

232 that the mean and standard deviation of the intensity values are zero and one,

233 respectively. Thus, the ACF makes no distinction between the phases. Visually, we

234 might consider the white phases to be the background or the matrix in Fig. 4, but the

235 ACF is only sensitive to the contrast between the phases and the relative abundance

236 of the phases.

238 The first example (Fig. 4a) has a single circle, which can be viewed as a "dilute

239 phase", in that the black circle occupies a very small area in the total aggregate. This

240 example is analogous to sparse amygdules or phenocrysts in a volcanic rock. The

241 ACF for this example ("Single Circle" in Fig. 4d) starts with $\rho=1$ at $r=0$ and

242 decreases to zero at $r=2 R$ where $R$ is the radius of the circle. The reason is that the

243 black phase is dilute enough that the only correlation is "self-correlation", where

244 objects still overlap with their offset copies.

246 The second example (Fig. 4b) has a square lattice of periodically repeating circles,

247 arranged with a uniform distance $4 R$ between the centers of each circle in the

248 horizontal and vertical directions. The ACF for this example is shown only for the

249 image horizontal direction ("Square Lattice" in Fig. 4d). This example has two

250 important differences from the "Singe Circle" example. First, the circles in this

251 example compose a much greater fraction of the image. As a result, the black circles

252 and white background have opposite signs in the rescaled image, and have negative

253 autocorrelation values where the circles and matrix overlap. This anticorrelation is 
254 expressed by the shorter decay lengthscale of the "Square Lattice" ACF compared to

255 the "Single Circle" ACF, and by the negative autocorrelation values near $r=2 R$ and $256 r=6 R$.

258 Second, the "Square Lattice" ACF also has additional maxima at $r=4 n R$, where

$259 n=1,2, \ldots \infty$ (Fig. $4 \mathrm{~d}$ shows the first of these maxima at $r=4 R$ ). These maxima

260 correspond to "neighbor-correlation" caused by overlap between neighboring

261 circles (c.f., blocking-function, Torquato, 2002). For this example, the self-

262 correlation and neighbor-correlation parts of the ACF are well separated because

263 the lattice spacing, $4 R$, is much larger than the diameter, $2 R$, of the circles.

265 The third example (Fig. 4c) has an irregular set of anticlustered circles, which are

266 meant to represent grains in a rock. Natural grains have an irregular (i.e., non-

267 periodic) arrangement and are anticlustered, meaning they do not physically

268 overlap. In addition, the shapes and placement of the objects are statistically

269 isotropic, meaning that there is no significant dependence on direction. Thus, the

270 ACF ("Anticlustered" in Fig. 4d) is solely dependent on the offset distance, $r=|\mathbf{r}|$.

272 The ACF for this example shows a shorter decay lengthscale at short lags $(r<R)$

273 when compared to the previous examples. As before, this results from

274 anticorrelation between the black circles and white background. This example also

275 shows a decaying periodic pattern at long lags $(r>R)$. The ACF is similar to that for

276 the Square-Lattice example, except for the fact that the self-correlation and 
277 neighbor-correlation parts of the ACF now overlap. This overlapping is expected in

278 natural geologic materials, where the spacing between grain centers is similar to the

279 diameter of the grains.

280

281 The anticlustered condition creates a decaying periodicity in the ACF, with a

282 wavelength proportional to the diameter of the circles. In statistical mechanics, this

283 problem is commonly treated using the pair-correlation function (Debye et al, 1957;

284 Torquato and Stell, 1982; Torquato and Stell, 1983; Torquato and Stell, 1985). The

285 pair-correlation function gives the probability of finding a particle center at a lag $r$

286 from a reference particle center. The density of points in a Fry diagram (Fry, 1979)

287 is proportional to the probability provided by the pair-correlation function. Note

288 that the peak at $\frac{r}{R}=2.5$ in the "Anticlustered" curve in Fig. $4 \mathrm{~d}$ corresponds to the

289 high-density girdle in the nearest-neighbor distribution of a Fry plot (Fry, 1979;

290 Panozzo Heilbronner, 1992). Torquato (2002) has a detailed review of this topic.

292 In the Introduction, we mentioned that there are cases where a material might lack

293 a spatially organized fabric. The next two examples highlight end-member cases.

294 The examples are so simple that illustrations are not needed. The first is a material

295 with only one phase. The image would have a uniform intensity, so $\rho$ would be

296 everywhere 1, regardless of the lag. This example is analogous to a monomineralic

297 rock. Of course, a monomineralic rock might be composed of grains with different

298 crystal orientations, as determined, for example, by electron backscatter diffraction.

299 For this case, the "phases" could be defined on the basis of orientation, and a 
300 standard ACF analysis would then be possible. The second example is a two-phase

301 material, where one phase is distributed randomly as infinitesimal grains. In

302 practical terms, infinitesimal would mean a grain that is near the size an individual

303 pixel or voxel. For this example, $\rho=1$ at $r=0$, but drops to $\rho=0$ for $r>0$.

305 We now consider the effects of deformation on autocorrelation. The ACF contains

306 information about both particle shapes and neighbor distances, and the focus here is

307 to distinguish between the deformation of particles, as measured by the Ramsay's

308 (1967) $R_{f} / \phi$ method, and the deformation of nearest-neighbor distances, as

309 measured by the Fry (1979) method.

310

311 For the first example (Fig. 5a-b), consider a 2D aggregate composed of regularly

312 spaced black ellipses with the short axis $R_{Y}$ equal to half the length of the long axis

$313 R_{X}$. The spacing is isotropic, meaning that the distances between ellipse centers is

314 the same in both the horizontal and vertical directions. The influence of anisotropy

315 on the ACF is indicated by different decay length scales: $\rho$ reaches zero at lag

$316 r / R_{Y}=2$ for the $Y$ direction, and at $r / R_{Y}=4$ for the $X$ direction. The ratio of these

317 two decay length scales is equal to the axial ratio of the ellipses. We use the term

318 "Ramsay effect" to describe this anisotropy of $\rho$ associated with self-correlation of

319 particle shapes. The ACFs for the $X$ and $Y$ directions show a second maxima at the

320 same lag, $r / R_{Y}=8$, which means that the neighbor correlation is isotropic. The Fry

321 method would show zero strain for this case. 
323 Figure 5 here.

325 Now consider the second anisotropic example (Fig. 5c-d), where circles with radius

$326 R$ are arranged on an anisotropic grid, such that the object centers are spaced $6 R$

327 apart in the $X$ direction, and $4 R$ in the $Y$ direction. The ACF is isotropic for self-

328 correlation. In other words, the length scale for initial decay of $\rho$ is the same,

329 regardless of orientation. Ramsay's $R_{f} / \phi$ method would find zero strain for this

330 example. Note, however, that $\rho$ increases back to one at $r / R=6$ in the $X$ direction

331 and $r / R=4$ in the $Y$ direction. In other words, $\rho$ is isotropic for self-correlation but

332 anisotropic for neighbor-correlation. Thus, we use the term "Fry effect" to describe

333 the anisotropy of $\rho$ due to the distribution of neighboring grains.

335 The last of our simple examples (Fig. 6) is a 2D aggregate made up of three phases,

336 rendered in black, gray, and white, and assigned image intensities of 10, 1, and 0 ,

337 respectively. The objective is to show how the ACF is affected when more than two

338 phases are present. The first example (Fig. 6a-b) has large black elliptical grains

339 with vertical long axes, and small gray elliptical grains with horizontal long axes.

340 The full 2D ACF is shown as contours of $\rho$ as a function of the $x$ and $y$ components of

341 the lag vector. All of the $\rho$ contours have the same elliptical shape as the black

342 grains. The reason is that black grains are large in size and have a large contrast

343 with the other intensities, so the ACF is strongly weighted towards the grain shapes

344 for this phase. 
346 The second example (Fig. 6c-d) shows what happens when the gray and black

347 phases are exchanged. The black elliptical grains are now small, but they still have a

348 strong contrast relative to the other two phases. The 2D ACF shows that, at short

349 lags, the $\rho$ contours have an elliptical shape similar to that for the black grains. As

350 the lag distance increases, the $\rho$ contours transition to an elliptical shape similar to

351 that of the large gray grains. The gray grains are larger in this example, which

352 compensates for its low contrast relative to the white phase, but only at long

353 lengthscales.

354

355 Figure 6 here.

356

357 This example highlights a subtle feature of autocorrelation for images with a range

358 of grayscale intensities. The ACF is more heavily weighted towards features that

359 have a strong contrast relative to the rest of the image. As a result, image processing

360 methods can be used to weight the ACF towards specific features of the fabric.

361 Useful methods potentially include gamma correction or image segmentation (e.g.

362 Barrett and Heilbronner, 2013). Remember, however, that the image is standardized

363 before calculating the ACF. As a result, the ACF is unaffected by linear rescaling of

364 the image intensities (Panozzo Heilbronner, 1992).

365

366 4. Estimating Grain Size in the ACF

367 Grain size is a significant parameter in its own right, but it is especially important

368 for distinguishing between self-correlation and neighbor-correlation. There are 
369 several ways to estimate the average grain radius. To guide this discussion, we

370 generated a 3D aggregate composed of non-overlapping, close-packed, identical

371 black spheres set in a white matrix (Fig. 7a). The circles vary in size, but this result is

372 simply due to sectioning through the 3D spheres, which otherwise have a uniform

373 radius $R=1$. The ACF for the full 3D volume as a function of radial lag exhibits both

374 self-correlation and neighbor-correlation (Fig. 7b). The spheres in this example

375 compose $54 \%$ of the volume.

376

377 Debye and Beuche (1949) proposed an exponential model for the ACF as

378 determined from X-ray scattering of atomic-scale vacancies

$$
\rho(r)=\exp \left(-r / R_{\mathrm{C}}\right)
$$

380 where $R_{\mathrm{C}}$ is the characteristic lag for the decay of $\rho$ with increasing $r$. Mätzler (2002)

381 showed that the characteristic lag, $R_{C}$ of the ACF for an aggregate of identical

382 overlapping spheres is equal to the radius of the spheres. Debye et al. (1957)

383 estimated $R_{C}$ using the derivative of the ACF at $r=0$. For (2), this estimate is

$384 R_{C} \approx-\left(\frac{\mathrm{d} \rho(r=0)}{\mathrm{d} r}\right)^{-1}$. The other alternative is to estimate the parameters in (2) using

385 a least-squares fit to the ACF (Mätzler, 2002). Neither approach is satisfactory. The

386 derivative method emphasizes only a small fraction of the ACF, and the least-

387 squares approach has problems separating self-correlation from neighbor-

388 correlation. This point is illustrated in Fig. 7b, which shows the poor fit of the

389 exponential model to the ACF of the simulated aggregate. The best-fit estimate $R_{C}=$

$390 \quad 0.31$ is much smaller than the expected value, $R=1$. 
392 A different approach approximates the grain size by using a characteristic point in

393 the short-lag part of the ACF (Panozzo Heilbronner, 1992; Buscombe et al., 2010).

394 The convention is to use the "half-height lag", $r^{*}$, which is the lag where $\rho$ first drops

395 to 0.5. Panozzo Heibronner (1992) estimated that the average grain radius is given

396 by $\bar{R} \approx 1.5 r^{*}$. Based on extensive calibration between the ACF and measurements

397 for images of unconsolidated sediments, Buscombe et al. (2010) recommended that

$398 \bar{R} \approx \pi r^{*}$.

399

400 Another approach to estimating grain size is the spherical model, which refers to the

401 idealized ACF for self-correlation of a single sphere (p. 79 in Torquato, 2002)

402

$$
\rho(r)=(r \leq 2 R)\left(1-\frac{3}{4} \frac{r}{R}+\frac{1}{16}\left(\frac{r}{R}\right)^{3}\right)
$$

403 Note that the logical expression $(r \leq 2 R)$ equals 1 if true and 0 if false. Substitution

404 of $\rho\left(r^{*}\right)=0.5$ into (3) gives

405

$$
\bar{R}=1.44 r^{*}
$$

406 which is nearly identical to the estimate of Panozzo Heibronner (1992).

408 In our experience, all of these approximations work poorly for dense aggregates. As

409 an example, the simulated aggregate has $r^{*}=0.35$. Thus, the approximations above

410 predict average grain radii of $0.50,0.53$, and 1.10 . These approximations are crude,

411 in part because they do not account for neighbor-correlation. The method suggested

412 by Buscombe et al., (2010) gives the closest approximation to the true value of one.

413 This is probably because our grain-supported aggregrate is similar to the 
414 unconsolidated sediments on which the recommendation is based. Buscombe et al.'s

415 method will diverge from the true radius for materials that cannot be approximated

416 as unconsolidated sediments.

418 Figure 7 here.

420 To overcome these issues, we propose a generalized two-component model for the 421 isotropic ACF

$$
\rho(r)=\left(r \leq 2 R_{\mathrm{S}}\right)\left(1-\frac{3}{4} \frac{r}{R_{\mathrm{S}}}+\frac{1}{16}\left(\frac{r}{R_{\mathrm{S}}}\right)^{3}\right)+f_{\mathrm{N}} \exp \left(-\left(\frac{r}{R_{\mathrm{N}}}\right)^{\beta}\right) \sin \left(\pi \frac{r}{R_{\mathrm{N}}}\right)
$$

423 where the self-correlation component of the ACF is represented by (3) and the

424 neighbor-correlation component, by a sinusoid with a decaying amplitude (modified

425 from equation 7 in Cule and Torquato, 1999). This model has four unknown

426 parameters: $R_{S}, f_{N}, R_{N}$ and $\beta$. $R_{\mathrm{S}}$ represents the average grain radius, and can be

427 viewed as the length scale for self-correlation. $f_{\mathrm{N}}$ defines the amplitude of the

428 neighbor component. Note that $f_{N}$ can be negative for multi-phase aggregates, given

429 that neighboring phases are likely to have different intensity values. The neighbor

430 component has two parts. The decay of the neighbor-correlation with $r$ is

431 represented by a stretched exponential function, where $R_{\mathrm{N}}$ defines the decay length

432 scale and the power $\beta$ defines the amount of stretching of the exponential function.

433 We have found that $\beta$ values other than one are needed to ensure a good fit to an

434 isotropic ACF. The periodicity for the neighbor-correlation is represented by the sin,

435 which has a wavelength of $2 R_{\mathrm{N}}$. Toquato (2002, Chapter 5) discussed a variety of 
436 analytical solution for ACFs for simple aggregates. Equation (5) does include the

437 spherical model for self-correlation, but it is otherwise empirical in its derivation.

438 The big advantage is that the two-component model is relatively simple and thus

439 much easier to use for estimation.

441 The unknown parameters for (5) can be estimated using a nonlinear least-squares

442 routine, such as the one discussed below for strain estimation. We have found that

443 the analysis works best by using the Fisher transform to convert $\rho$ to $\zeta$, which has

444 more uniform variance with $r$ (see below for details). The starting estimates for $R_{\mathrm{S}}$

445 and $R_{\mathrm{N}}$ are set to be about five times larger than $\bar{R}$, as determined from (4). This

446 strategy is important for avoiding local minima for the best-fit solution. Starting

447 estimates for $\beta$ and $f_{\mathrm{N}}$ are set to 1 and 0 , respectively. $R_{\mathrm{S}}$ and $R_{\mathrm{N}}$ are constrained to

448 be positive.

450 The two-component model gives a best-fit solution for the synthetic example of $R_{\mathrm{S}}=$

451 0.97, which compares well with the true value of 1 . The estimates for the other

452 parameters are $f_{\mathrm{N}}=-0.36, R_{\mathrm{N}}=1.89$, and $\beta=3.41$. The fit has an $R^{2}=0.82$. We use

453 the F ratio test of Bevington and Robinson (2003, p. 207) to compare the fit

454 provided by the two-component model and the spherical model. In all cases, the

455 two-component model provides a significantly better fit.

457 Fig. 7c shows the best-fit ACF, along with the predicted components for self-

458 correlation and neighbor-correlation. The ACF is dominated by the self-correlation 
459 component, but both components are significant at short lags, $0<r / R<2$. The

460 neighbor-correlation component commonly decays to zero by $r / R=5$. Thus there is

461 little need to analyze the ACF beyond that point.

462

\section{$463 \quad$ 5. Estimating the Autocorrelation Function}

464 Consider the general case for an image, where $U(x, y, z)$ are continuously varying

465 scalar values within a 3D domain spanned by $x, y$, and $z$. We focus here on X-ray

466 attenuation, but the scalar field can be anything that characterizes the material in

467 that domain. Our objective is to estimate the autocorrelation for this field variable as

468 a function of the components of the lag vector, $r_{x}, r_{y}$, and $r_{z}$. We introduce a

469 standardized variable $U_{\mathrm{s}}$ defined by

470

$$
U_{\mathrm{s}}=\frac{U-c}{\sigma(U-c)}
$$

471 where $c$ is the mean value for $U$ and $\sigma(U-c)$ is the standard deviation of $U$ around

472 the mean. The standardization in (6) ensures that $\rho$ remains within the limits of \pm 1 .

473 The ACF is defined using $U_{\mathrm{s}}$ (Priestley, 1981),

$$
\rho\left(r_{x}, r_{y}, r_{z}\right)=\iiint_{-\infty}^{\infty} U_{\mathrm{s}}(x, y, z) U_{\mathrm{s}}\left(x-r_{x}, y-r_{y}, z-r_{z}\right) \mathrm{d} x \mathrm{~d} y \mathrm{~d} z .
$$

475 The ACF is simply a cross-correlation of the field variable with a copy that is offset

476 by $r_{x}, r_{y}$, and $r_{z}$. Equation (5) provides a formal definition, but it is too cumbersome 477 to serve as a method of calculation. 
479 The Fourier transform provides a faster way to calculate the ACF. We start with a 480 discrete uniform grid of standardized attenuation values, $U_{s}\left(j_{x}, j_{y}, j_{z}\right)$, where $\Delta$ is the 481 grid spacing, and $j_{x}=0$ to $N_{x}-1, j_{y}=0$ to $N_{y}-1$, and $j_{z}=0$ to $N_{z}-1$ are space-

482 domain indices in the $x, y$, and $z$ directions The Fourier transform is used to convert 483 the grid into the wave domain. The Fourier coefficients are given by

$$
\mathcal{U}_{\mathrm{s}}\left(k_{x}, k_{y}, k_{z}\right)=\mathcal{F}\left(U_{\mathrm{s}}\left(j_{x}, j_{y}, j_{z}\right)\right)
$$

485 where $\mathcal{F}$ and $\mathcal{F}^{-1}$ are operators that indicate the Fourier transform and its inverse, 486 and $k_{x}=0$ to $N_{x}-1, k_{y}=0$ to $N_{y}-1$, and $k_{z}=0$ to $N_{z}-1$ are wave-domain 487 indices in the $x, y$, and $z$ directions. Keep in mind that the Fourier coefficients $488 u\left(k_{x}, k_{y}, k_{z}\right)$ are, in general, complex valued.

490 The Fourier coefficients of the ACF are determined by the products

$$
\mathcal{R}\left(k_{x}, k_{y}, k_{z}\right)=\mathcal{U}_{\mathrm{s}}\left(k_{x}, k_{y}, k_{z}\right) \mathcal{U}_{\mathrm{s}}^{*}\left(k_{x}, k_{y}, k_{z}\right)
$$

492 where * indicates the complex conjugate. The inverse Fourier transform is used to 493 return the ACF to the space domain,

$$
\rho\left(j_{x}, j_{y}, j_{z}\right)=\mathcal{F}^{-1}\left(\mathcal{R}\left(k_{x}, k_{y}, k_{z}\right)\right)
$$

496 Consider the following MATLAB example, where imageFile.tif is 2D grayscale image

497 file accessible in the current directory. The image should have an odd number of 498 pixels in each dimension.

499 1. $\mathrm{U}=$ double(imread('imageFile.tif'));

$500 \quad$ 2. $\quad \mathrm{c}=\operatorname{mean}(\mathrm{U}(:))$; 
3. $\mathrm{Us}=(\mathrm{U}-\mathrm{c}) / \operatorname{sqrt}\left(\operatorname{mean}\left((\mathrm{U}(:)-\mathrm{c}) \cdot^{\wedge} 2\right)\right)$;

502

4. $\mathrm{N}=\operatorname{numel}(\mathrm{U})$;

503

5. Uscript $=f f t n(U s) / N$;

504

6. $\quad$ Rscript $=$ Uscript. ${ }^{*}$ conj(Uscript);

505

7. Rho $=$ fftshift(ifftn(Rscript $\left.)^{*} \mathrm{~N}\right)$;

506

8. $[\mathrm{nx}, \mathrm{ny}]=\operatorname{size}(\mathrm{U})$;

507

9. $\mathrm{rx}=$ linspace $(-(\mathrm{nx}-1) / 2,(\mathrm{nx}-1) / 2, \mathrm{nx})$;

508

10. ry $=$ linspace(-(ny-1)/2,(ny-1)/2,ny);

509

11. $[\mathrm{Ry}, \mathrm{Rx}]=\operatorname{ndgrid}(\mathrm{rx}, \mathrm{ry})$;

510

$$
\text { 12. } \mathrm{r}=\operatorname{sqrt}\left(R x .^{\wedge} 2+R y . \wedge 2\right) \text {; }
$$

511 13. figure; $\operatorname{plot}(\mathrm{r}(:)$, Rho(:), '.');

512 14. figure; pcolor(Rx, Ry, Rho); shading interp; axis equal;

513 Line 1 loads the image into the matrix $\mathrm{U}$ and converts the values to double precision.

514 Lines 2 and 3 standardize the matrix. Lines 4-7 calculate the ACF, and lines 8-12

515 calculate the $x$ and $y$ lag components in units of pixels. Line 13 plots the ACF as a

516 function of lag distance. Line 14 plots the ACF as a 2D color map. Note that the

517 "fftshift" command in line 7 organizes the matrix Rho so that the zero lag, $r=0$, is

518 located at the central voxel.

519

520 There has been a lot of discussion about how to reduce bias and increase precision

521 when estimating the ACF (e.g., Priestley, 1981; Broerson, 2006). For our study of

522 fabrics, we are not as interested in the true values of the autocorrelation, but rather

523 the anisotropy of the autocorrelation. As such, we have found that the Fourier 
524 method, which has been criticized as less than optimal for estimating the true values

525 of the ACF (Broerson, 2006), does the best job preserving sample anisotropy.

526 Windowing functions are commonly used in conjunction with the finite Fourier

527 transform to reduce edge effects and enhance spectral resolution (e.g., Press et al.,

528 2007, p. 656-660). Our tests using various windowing filters have found that typical

529 windowing functions, such as the Hann filter, generate artificial anisotropy in the

530 sample, which causes biases in the estimated deformation parameters. Sample

531 anisotropy is best preserved by calculating the ACF from the untreated standardized

532 image. (Of course, the image has already been delimited (i.e., windowed) to finite

533 dimensions.) The edges of the image do generate some ringing (i.e., the Gibbs effect),

534 but this ringing appears to contribute relatively little to the ACF (also see Panozzo

535 Heilbronner, 1992).

536

537 6. Estimating Deformation Parameters

538 To estimate the deformation parameters from a sample ACF, we search for a stretch

539 tensor that reduces the ACF to an isotropic form. The assumption, one that is used in

540 almost all strain methods, is that the fabric of the material was isotropic before

541 deformation.

542

543 The deformation of a material line is defined by

544

$$
\mathbf{r}=\mathbf{V R r}_{0}
$$

545 where $\mathbf{V}$ is the left-stretch tensor, $\mathbf{R}$ is a rotation tensor, and $\mathbf{r}_{0}$ and $\mathbf{r}$ are vectors

546 representing the material line before and after the deformation. For geologic 
547 studies, there is generally little information about $\mathbf{R}$. Thus, the estimation is focused

548 solely on the stretch tensor. The left-stretch tensor representation is preferred

549 because it can be calculated without knowing $\mathbf{R}$. The stretch tensor is usually

550 decomposed into its principal form

$$
\mathbf{V}=\mathbf{T} \boldsymbol{\Lambda}_{\mathbf{V}} \mathbf{T}^{\mathrm{T}}
$$

552 where $\mathbf{T}$ is the transformation matrix with column vectors that correspond to the

553 principal stretch axes in the sample coordinate frame. The superscript $\mathrm{T}$ indicates

554 the matrix transpose. The tensor $\Lambda_{\mathrm{V}}$ is diagonalized, with the principal stretches

$555 S_{X}, S_{Y}$, and $S_{Z}$ on the diagonal and zeros elsewhere else. The Hencky tensor, E,

556 defined by

$$
\begin{gathered}
\mathbf{V}=\exp (\mathbf{E})=\mathbf{T} \exp \left(\boldsymbol{\Lambda}_{\mathbf{E}}\right) \mathbf{T}^{\mathrm{T}} \\
\mathbf{E}=\ln (\mathbf{V})=\mathbf{T} \ln \left(\boldsymbol{\Lambda}_{\mathbf{V}}\right) \mathbf{T}^{\mathrm{T}} \text { and }
\end{gathered}
$$

559 provides a better formulation for statistical estimation of the stretch tensor

560 (Brandon, 1995; Mookerjee and Peek, 2014). Like the stretch tensor V, the Hencky

561 tensor is symmetric, which means that it has six unique values, as indicated by

$562 \mathbf{E}=\mathbf{E}^{\mathrm{T}}$. The transformation matrix is the same for both $\mathbf{V}$ and $\mathbf{E} . \boldsymbol{\Lambda}_{\mathbf{E}}$ is a diagonal

563 matrix with the principal natural stretches $E_{X}, E_{Y}, E_{Z}$ on the diagonal. The expanded

564 versions on the right side of $(13 a, b)$ use the matrix definitions for the natural

565 logarithm and exponential functions (Higham, 2008). The natural volume-strain is

$$
E_{V}=\ln \left(V / V_{0}\right)=\operatorname{tr}(\mathbf{E}),
$$

567 where $V_{0}$ and $V$ are the initial and final volume of the sample, and tr is the trace (i.e.

568 sum of the diagonal elements). The deviatoric Hencky tensor is defined by

$$
\mathbf{E}^{\prime}=\mathbf{E}-\mathbf{I} E_{V} / 3
$$


570 where I is the identity matrix. Most methods of strain analysis do not provide

571 information about the volume-strain component of the deformation (Brandon,

572 1995). This is also true for autocorrelation estimates of strain, given that we have no

573 information about the length scale of the ACF for the material prior to deformation.

574 In some cases, one can make independent estimates of volume strain (Wright and

575 Platt, 1982; Ring and Brandon, 1999). Substitution of this information into (15) can

576 be used to convert $\mathbf{E}^{\prime}$ to $\mathbf{E}$.

577

578 We follow the usual convention where the principal values are organized as

$579 E_{X} \geq E_{Y} \geq E_{Z}$, referring to the maximum extension direction $X$, the intermediate

580 direction $Y$, and the maximum shortening direction $Z$.

581

582 The Hencky tensor extends the scalar concept of natural strain, $E=\ln (S)$, to a

583 tensor representation, where stretch $S=l / l_{0}$ with $l$ and $l_{0}$ representing the initial

584 and final lengths of a material line. As proposed by Hencky (1931), natural strain

585 scales linearly with strain rate during a finite deformation, so it provides a more

586 natural representation of strain. In addition, natural strain also allows for a simpler

587 representation of errors, as symmetric Gaussian variations around a mean value. In

588 fact, the natural $\log$ is one of several power transforms that are used in statistics to

589 transform data sets into a form where standard Gaussian statistics can be used (Box

590 and Cox, 1964).

591 
592 For our purposes, we need to specify the change in the lag vector from its present

593 deformed state to its initial undeformed state. The ACF is assumed to have been

594 isotropic in the initial state, so we only need the magnitude $r_{0}$ of the initial lag vector

$595 r_{0}=\left\|\mathbf{r}_{\mathbf{0}}\right\|$. We define an "undeforming" function $f_{\mathrm{u}}$ (c.f. Spencer, 1980, eq. 6.21),

$$
r_{0}=f_{\mathrm{u}}\left(\mathbf{r} ; \mathbf{E}^{\prime}\right)=\sqrt{\mathbf{r}^{\mathrm{T}} \mathbf{V}^{\prime}-2} \mathbf{r}=\sqrt{\mathbf{r}^{\mathrm{T}} \exp \left(-2 \mathbf{E}^{\prime}\right) \mathbf{r}} .
$$

597 Note that in this equation and elsewhere below, we use a semicolon to separate

598 arguments on the left (e.g., r), from parameters on the right (e.g., E'). Arguments are

599 given values, and parameters are values to be estimated.

600

\section{$601 \quad 6.1$ Estimating the Initial Autocorrelation Function}

602 Autocorrelation is limited to the range -1 to +1 , which makes the variable poorly

603 suited for statistical analysis. The Fisher transform $\zeta=\operatorname{arctanh}(\rho)$ and its inverse

$604 \rho=\tanh (\zeta)$ link the autocorrelation variable $\rho$ to a new variable $\zeta$, which has a

605 range $-\infty$ to $+\infty$. This variable $\zeta$ usually follows a Gaussian distribution

606 (Alexander, 1997), which makes it better suited for least-squares estimation,

607 including calculation of uncertainties for the estimated parameters.

608

609 We seek a least-squares estimate for the unknown parameters $\mathbf{E}^{\prime}$ and $\boldsymbol{c}$. We have

610 already introduced $\mathbf{E}^{\prime}$ above, which contains 6 unique coefficients. The vector $\boldsymbol{c}$

611 contains coefficients for a spline, which is used to represent the isotropic ACF as a

612 function of $r_{0}$, as represented by $\zeta_{0}=\zeta_{0}\left(r_{0} ; \boldsymbol{c}\right)$. We have experimented with several

613 types of functional forms for $\zeta_{0}$, but we found that a cubic spline is both sufficiently 
614 flexible to represent the isotropic ACF for all of our test cases and computationally

615 fast, as needed for a nonlinear search algorithm. We would prefer to use the two-

616 component model (5) but it is computationally too slow for our estimation here. The

617 two-component model is used afterward to find best-fit estimates for the

618 undeformed ACF.

619

620 For our least-squares calculation, we convert the gridded problem above into a

621 serial list of $i=1$ to $n$ model equations

$$
\zeta_{i}=\zeta_{0, i}\left(f_{u}\left(\mathbf{r}_{i} ; \mathbf{E}^{\prime}\right) ; \boldsymbol{c}\right)+\varepsilon_{i}
$$

623 where $n=N_{x} N_{y} N_{z}$. Equation (17) shows that we are using the estimated isotropic

624 ACF, along with the undeformed length of $\mathbf{r}$, to get predicted autocorrelation values.

$625 \varepsilon_{i}$ is a stochastic variable, and represents errors associated with the autocorrelation

626 measurement. $\varepsilon_{i}$ is assumed to be independently and identically distributed, with an

627 expected mean of zero, and a variance of $\sigma_{\varepsilon}^{2}$.

628

629 The best-fit estimates $\hat{\mathbf{E}}^{\prime}$ and $\hat{\boldsymbol{c}}$ are found numerically by searching for a candidate 630 solution that minimizes the objective function,

$$
\tilde{S}=S\left(\tilde{\mathbf{E}}^{\prime}, \tilde{\boldsymbol{c}}\right)=\frac{1}{v} \sum_{i=1}^{n}\left[\zeta\left(\mathbf{r}_{i}\right)-\zeta_{0}\left(f_{\mathrm{u}}\left(\mathbf{r}_{i} ; \tilde{\mathbf{E}}^{\prime}\right) ; \tilde{\boldsymbol{c}}\right)\right]^{2}
$$

632 The tilde and caret symbols are used to indicate the state of estimation for a

633 parameter variable. The tilde indicates a candidate estimate in the search, and the

634 caret indicates a best-fit estimate that minimizes the least-squares objective 635 function. 
637 The degrees of freedom, $v$, for the objective function is defined by $v=n-m_{\mathbf{c}}-m_{\mathbf{E}}$, 638 where $m_{\mathbf{c}}$ and $m_{\mathbf{E}}$ are the number of unknown parameters in $\boldsymbol{c}$ and $\mathbf{E}$. The best-fit 639 solution provides an estimate for $\hat{\sigma}_{\varepsilon}^{2}=\hat{S}$.

641 Equation (18) is a separable least-squares problem (Moler, 2004, p 154), in that the 642 piecewise-spline estimate has a linear least-squares solution and the strain-tensor 643 estimate has a nonlinear least-squares solution. As a result, the nonlinear search is 644 focused exclusively on estimating the strain tensor. We use the "fmincon" function 645 in MATLAB to estimate the six coefficients of the Hencky tensor

$$
\mathbf{E}^{\prime}=\left[\begin{array}{lll}
E_{11}^{\prime} & E_{12}^{\prime} & E_{13}^{\prime} \\
E_{12}^{\prime} & E_{22}^{\prime} & E_{23}^{\prime} \\
E_{13}^{\prime} & E_{23}^{\prime} & E_{33}^{\prime}
\end{array}\right],
$$

647 subject to the constraint $\operatorname{tr}\left(\mathbf{E}^{\prime}\right)=E_{11}^{\prime}+E_{22}^{\prime}+E_{33}^{\prime}=0$. Thus, the effective number of 648 unknown coefficients for $\mathbf{E}^{\prime}$ is $m_{\mathbf{E}}=5$ for a 3D data set. Note that (16-19) are easily 649 adapted to estimate a 2D strain tensor, which would have $m_{\mathbf{E}}=2$.

651 Equation (18) is minimized in the following manner:

652 1) The search is started with a candidate solution $\tilde{\mathbf{E}}^{\prime}=0$, which corresponds to 653 zero deformation.

654 2) Equation (14) and $\tilde{\mathbf{E}}^{\prime}$ are used to convert the observed lag vectors $\mathbf{r}_{\mathrm{i}}$ to a 655 candidate estimate of their initial lag distances, $\tilde{r}_{0, i}$. 
656 3) A candidate estimate for the initial isotropic autocorrelation function,

$657 \zeta_{0}\left(f_{u}\left(\mathbf{r}_{i} ; \tilde{\mathbf{E}}^{\prime}\right) ; \widetilde{\boldsymbol{c}}\right)$, is estimated using a spline as a model function with $\tilde{r}_{0, i}$, and $\zeta_{i}$.

658 4) The objective function (18) is evaluated using the candidate estimate

$659 \quad \zeta_{0}\left(f\left(\mathbf{r}_{i} ; \tilde{\mathbf{E}}^{\prime}\right) ; \widetilde{\boldsymbol{c}}\right)$.

660 5) The value of the objective function for the candidate estimates, $\tilde{S}\left(\tilde{\mathbf{E}}^{\prime}, \widetilde{\boldsymbol{c}}\right)$ is passed

661 to the nonlinear search-algorithm, which tests for convergence to a final

662 minimum value for $\tilde{S}$. If the test is positive, $\tilde{S}$ is returned as the best-fit estimate,

$663 \hat{S}$, and the routine also returns the best-fit estimates for the parameters, $\hat{\mathbf{E}}^{\prime}$ and $\hat{\boldsymbol{c}}$.

664 If the test is negative, then the algorithm selects a new candidate estimate for

$665 \quad \tilde{\mathbf{E}}^{\prime}$ and returns to step 2 to evaluate that estimate.

666

667 With any nonlinear problem, there is always a concern that the optimization will

668 become trapped in a local minimum, resulting in a flawed estimate. Our algorithm

669 shows no sign of this kind of problem. Perhaps most telling is that the optimization

670 results in estimates that fit our different datasets very well. Thus, we suspect that

671 the objective function has a well-defined global minimum near the starting point

$672 \quad \tilde{\mathbf{E}}^{\prime}=0$. Bootstrap experiments, discussed below, indicate that the objective function

673 has a quasi-Gaussian form around the best-fit minimum. There may be future cases

674 where the solution fails, but this should be easy to spot by a poor fit between the

675 best-fit objective function and data, or by wild, non-physical estimates for the

676 parameters and their uncertainties.

677

$678 \quad 6.2$ Assessment of Fit 
679 The quality of fit can also be judged using a residuals plot, where the standardized

680 residuals $\varepsilon_{i} / \sigma_{\varepsilon}$ are plotted on the $y$-axis and the estimated initial lag distance $r_{0, i}$, on

681 the $x$-axis. The standardized residuals should be independently and identically

682 distributed with a mean of zero and a standard deviation of one.

683

684 Another useful measure of the fit is the $R^{2}$ statistic, which is defined using the

685 conventional formula

686

$$
R^{2}=1-\frac{\operatorname{var}\left(\varepsilon_{i}\right)}{\operatorname{var}\left(\zeta_{i}\right)}
$$

687

688 The statistical independence of the residuals is tested using the Durbin-Watson

689 statistic $d$ (p. 68, Draper and Smith, 1998),

690

$$
d=\frac{\sum_{j=2}^{n}\left(\varepsilon_{j}-\varepsilon_{j-1}\right)^{2}}{\sum_{j=2}^{n} \varepsilon_{j}^{2}}
$$

691 where the subscript $j$ indicates the residuals in order of increasing $r_{0}$. The null case,

692 where there is no serial correlation, has an expectation $d=2$.

693

\section{$694 \quad 6.3$ Uncertainties for Estimated Strain Parameters}

695 At termination, the MATLAB function "fmincon" reports the Hessian matrix $\mathbf{H}$ for

696 the best-fit solution $\mathbf{E}^{\prime}$. The Hessian is used to derive a linear approximation to the

697 parameter covariance matrix (Press et al., 2007, p. 799-802)

698

$$
\operatorname{cov}\left(\operatorname{vech}\left(\mathbf{E}^{\prime}\right)\right) \approx \sigma_{\epsilon}^{2} \mathbf{H}^{-1}
$$


699 where the half-vectorization function, vech, converts $\mathbf{E}^{\prime}$ into a six-component

700 column vector for the strain parameters. As a result, $\operatorname{cov}\left(\operatorname{vech}\left(\mathbf{E}^{\prime}\right)\right)$ is a $6 \times 6$

701 covariance matrix for the six strain parameters in $\mathbf{E}^{\prime}$.

702

703 For a nonlinear inverse, there is also a concern that approximated uncertainties may

704 contain large errors. In a test, we found that uncertainties approximated by (22)

705 agreed well with more accurate estimates determined by the bootstrap method

706 (Efron, 1979). This result suggests that, near the best-fit solution, $S$ has a quasi-

707 linear relationship with respect to the parameters (Press et al., 2007, p. 807-818).

708 We could use the bootstrap method to estimate all errors, but it is computationally

709 expensive.

710

711 Given uncertainties for the coefficients in $\mathbf{E}^{\prime}$, it is a simple matter to use the method

712 of Hext (1963) to calculate uncertainties for the principal values and principal

713 directions. This method has been used in the Yale STRAIN3D program since 1996,

714 and works well for estimating uncertainties for strain analysis. The method is also

715 widely used for statistical analysis of anisotropic magnetic susceptibility data (e.g.,

716 Hext, 1963; Jelinek, 1978; Owens, 2000).

717

718 We report 95 percent confidence intervals for both the estimated magnitudes and

719 directions for the principal strains, and the isotropy test of Hext (1963), which tests

720 if the principal strains are significantly different from each other. The uncertainty

721 for a best-fit principal direction is represented by an elliptical cone that defines the 
72295 percent confidence limit for the estimated principal direction (Fig. 8). The

723 orientation of each elliptical cone is reported as the twist, which is the azimuth of

724 the long axis of the cone in the plane normal to the best-fit principal direction. Keep

725 in mind that principal directions are orthogonal. So given a best-fit principal

726 direction, the twist is defined by the azimuth from the second principal direction to

727 the third principal direction. The order of the directions is defined by the repeating

728 sequence $+X,+Y$, and $+Z$, which are positive axes in a right-handed coordinate frame.

729 For example, a twist of +10 degrees for the long-axis of the $\alpha_{95}$ elliptical cone

730 around the best-fit estimate for the $Y$ principal-strain direction, would lie at 10

731 degrees from the $+Z$ direction towards the $+X$ direction. For most cases, the twist is

7320 or 90 degrees, which indicates that the elliptical cone is aligned with the second

733 and third principal directions.

734

735 Figure 8 here.

736

737 7. Strain Estimation Examples

738 To demonstrate the advantages and limitations of our method, we find the best-fit

739 strain parameters for several examples. The first set of examples, called "phantoms"

740 (e.g., Shepp and Logan, 1974; Ketcham, 2005b), are synthetic cases where numerical

741 calculations are used to generate an isotropic aggregate of spherical grains, which is

742 then subjected to a known homogeneous deformation. The second set of examples

743 illustrates estimation for naturally deformed rocks. 


\section{$745 \quad 7.1$ 3D Synthetic (Phantom) Material}

746 A 3D isotropic aggregate of spherical grains was generated using an event-driven,

747 molecular-dynamics sphere-packing code (Lubachevsky and Stillinger, 1990; Donev

748 et al., 2005a; 2005b; Skoge et al., 2006; available at

749 http://cherrypit.princeton.edu/donev/Packing/PackLSD/Instructions.html). The

750 code begins with a set of randomly distributed spheres, each with an initial velocity.

751 The spheres interact with each other through collisions. The diameters of the

752 spheres grow linearly in time until the number of collisions diverges and the

753 diameters of spheres can no longer increase (see Donev, 2006 for an excellent

754 introduction to these algorithms). These simulations are routinely used to study

755 packing and flow in granular materials, (e.g., Torquato 2002; Luding, 2004). This

756 method generates aggregates that have the dense packing typical of geologic

757 aggregates. The algorithms can also be used to create phantom materials with

758 multiple grain sizes (Kansal et al., 2002; Donev et al., 2005a; 2005b), and non-

759 spherical grain shapes (Donev et al., 2005a; 2005b).

760

761 Figure 9 here.

762

763 The phantom contains 1200 non-overlapping grains that are packed to an average

764 porosity of 50 percent (Fig. 9a). Prior to deformation, the grains were identical

765 spheres, with a radius equal to 10 voxels. This dimension is set to $100 \mu \mathrm{m}$ for the

766 ACF calculation. The phantom was deformed coaxially with principal stretches $S_{X}=$

$7672, S_{Y}=1$, and $S_{Z}=0.5$. The $X$ direction was rotated 30 degrees from the sample $x$-axis 
768 toward the sample $y$-axis and the $Z$ direction was aligned with the sample $z$-axes.

769 The ACF for the deformed phantom is anisotropic, which causes a large spread in $\zeta$,

770 especially at $\frac{r}{R}<1.5$ (Fig. 9b). Once the deformation is removed using the estimated

771 best-fit strain parameters, the ACF collapses down to a single curve (Fig. 9c). The

772 best-fit parameters agree well with those for the known deformation (Table 1). Note

773 that the estimated grain radius, $R_{S}=97 \mu \mathrm{m}$, matches well with the known grain

774 radius, $100 \mu \mathrm{m}$. The standardized residuals show some structure, but otherwise

775 appear to be fairly uniform with increasing $r_{0} / R$ (Fig. 9d). The Durbin-Watson test

776 indicates $d=1.97$, which is consistent with our assumption that the residuals are

777 independent.

778

779 Table 1 here.

780

781 Table 1 shows estimated deviatoric principal strains in terms of both stretches $S$

782 and natural strains $E$, which are reported in centi-Neper (cNp). The Neper is the unit

783 of measure for the natural-log scale (Mills, 1995), in the same sense that the Bel is

784 the unit of measure for the base- $10 \log$ scale. A cNp is therefore equal to one

785 hundredth of a natural strain unit, in the same way that a dB is equal to one tenth of

786 a Bel. This scaling is convenient because, for small natural strains, in the range - 15

$787 \mathrm{cNp}<E<+15 \mathrm{cNp}$, the $\mathrm{cNp}$ designation is approximately equal to percent

788 elongation $\left(100 \Delta l / l_{0}\right)$. This relationship results from the small-value

789 approximation for the natural $\log , \ln (1+\epsilon) \approx \epsilon$ for $|\epsilon| \ll 1$. 790 
791 The confidence intervals for the stretches become asymmetric as the stretch

792 deviates from 1 , whereas the natural log confidence intervals remain symmetric in

793 all cases. We have shown above that the Hencky tensor, which is the tensor

794 representation of the natural strain, provides a compact notation relating tensor

795 properties to strain invariants (e.g., $E_{D}$ and $E_{V}$ in Brandon, 1995). Likewise, the

796 natural strains provide a simpler basis for estimating and reporting uncertainties.

797

$798 \quad 7.22$ D Image of Undeformed Oolite

799 Our.first natural example is a 2D photomicrograph of undeformed ooids from the 800 Jurassic Stump formation in Wyoming (Fig. 10; Crespi, 1986). The image is more

801 complicated than our previous examples, in that the ooids have internal structure

802 and varying sizes. The Ramsay effect for this image is visible in the initial decay of

803 the ACF. The Fry effect is small for three reasons. First, the ooids are anticlustered

804 (e.g., Fig. 4d), but they are otherwise randomly distributed. Second, the size

805 variation of the ooids reduces the Fry effect (e.g., Erlsev, 1988). Third, resolution of

806 the Fry effect requires averaging over a much larger set of ooids (Crespi, 1986), and

807 thus would require a larger image.

808

809 Figure 10 here.

810

811 For this example, the strain estimates given by the ACF method (Table 1) agree with

812 those from the $R_{f} / \phi$ method, which was calculated using outlines for 42 ooids and

813 the $R_{f} / \phi$ estimation algorithm of Shimamoto and Ikeda (1976). Note that for the 2D 
814 examples in Table 1, strains are reported as plane-strain deviatoric values given that

815 we have no information about volume strain or the out-of-plane strain. The high

816 value of $R^{2}$ indicates the best-fit solution fits the data very well. The Durbin-Watson

817 test indicates no significant serial correlation. The residuals plot (Fig. 9d) shows

818 some structure, but this is not unexpected given that autocorrelation estimates tend

819 to retain some serial correlation (Priestley, 1981; Broersen, 2006).

820

$821 \quad 7.32$ D Image of Deformed Oolite

822 A second photomicrograph example (Fig. 11a) shows deformed ironstone ooids

823 (figure 7.7 in Ramsay and Huber, 1983). This sample is interesting because the ooid

824 cores were deformed by an intracrystalline mechanism, whereas the rims show

825 truncations and fiber overgrowths, consistent with pressure solution. Ramsay and

826 Huber (1983) used the $R_{f} / \phi$ and "Nearest Neighbor" methods to estimate the

827 within-ooid strain and the bulk strain, respectively. The $R_{f} / \phi$ results were

828 determined by us (Table 1), using digitized outlines of laminae from the core of the

829 ooids, where they are not truncated by pressures.. The estimates are virtually

830 identical to those from Ramsay and Huber (1983, p. 120, axial ratio =1.22). The

831 "Nearest Neighbor" estimate is based on Fig. 7.18 from Ramsay and Huber (1983).

832 All three methods give the same principal directions. The ACF and $R_{f} / \phi$ estimates

833 are essentially identical (Table 1). The reason is that the ACF is weighted towards

834 the high-contrast ooid cores. The "Nearest Neighbor" estimate shows more strain,

835 but this is expected given the deformation between the grains, including dissolution 836 and overgrowths. 
838 Figure 11 here.

839

840 The ACF method works best where each grain has a relatively uniform intensity. The

841 concentric rings in the ooids could cause problems. The ACF is influenced by this

842 within-grain structure, which might cause the ACF method to give biased strain

843 estimates (Fig. 12). The 2D image was constructed by drawing two circular rings

844 and then deforming the rings to a strain ratio $\frac{s_{X}}{S_{Z}}=2$. At small lags, the ACF contours

845 for this image have simple elliptical shapes, but, at larger lags, the contours take on

846 "hot dog" shapes caused by smearing in the $X$ direction. The smearing results from

847 correlation between the different rings, which produces an upward bias in the strain

848 ratio $\frac{s_{X}}{S_{Z}}=2.1$, as estimated using the ACF method. At larger lags, the correlation

849 between the different rings is reduced, and the contour shape is more elliptical.

850

$851 \quad 7.4$ 3D Tomograms of Pressure-Solved Sandstones

852 We now consider X-ray attenuation tomograms for a pressure-solved sandstone

853 from the Olympic Subduction Complex in Washington State (Brandon et al., 1998).

854 Sample 92810-3 is from the Grand Valley Assemblage (Tabor and Cady, 1978) and

855 was deformed along a path that reached a maximum depth of about $12 \mathrm{~km}$ and a

856 maximum temperature of $250^{\circ} \mathrm{C}$ at about $18 \mathrm{Ma}$ (Brandon et al., 1998). The

857 microstructure of the sample is typical of pressure-solved sandstones from this and 
858 other subduction complexes (Tabor and Cady, 1978; Feehan and Brandon, 1999;

859 Ring and Brandon, 1999; Ring et al., 2001; Rahl et al., 2011).

860

861 The tomogram (Fig. 13) was acquired at the X-ray computed tomography facilities

862 at the Advanced Photon Source at Argonne National Labs (Beamline 13-BM-D). The

863 voxels are cubes, measuring $7.54 \mu \mathrm{m}$ on each side. The sample is approximately $3 \mathrm{x}$

$8643 \times 10 \mathrm{~mm}$, with $X$ approximately aligned with the long dimension of the sample. The

865 X-ray attenuation is shown by the gray scale, increasing from black to white.

866

867 Figure 13 here.

868

869 The sandstone is a turbidite and is dominated by angular, first-cycle volcanic

870 detritus. The tomogram shows features related to both the composition of the

871 sandstone and the fabrics resulting from the pressure-solution deformation (Fig.

872 14). As a reminder, X-ray attenuation increases with grain density and mean atomic

873 number. In particular, minerals with high concentrations of $\mathrm{Fe}, \mathrm{Mg}$, $\mathrm{Ti}$ and $\mathrm{Zr}$ are

874 bright (high attenuation). For this sample, the white grains correspond to pyrite,

875 magnetite and zircon; the intermediate grains, to lithic rock fragments; and the dark

876 grains, to quartz and plagioclase.

877

878 Figure 14 here.

879 
880 The tomogram also resolves fabrics related to the pressure-solution deformation.

881 Bright values on grain margins and selvages are related to residual concentrations

882 of insoluble phases, such as Fe-Ti oxides and phyllosilicates. The fiber overgrowths

883 are dominated by quartz and white mica, and have a fairly uniform appearance

884 when viewed in thin section, despite the heterogeneous assortment of detrital

885 grains. In the tomogram, the overgrowths also have a uniform appearance

886 throughout the volume. They everywhere seem to have a higher X-ray attenuation

887 than quartz, which might be due to the higher atomic number of potassium in the

888 white mica.

889

890 Table 2 here.

891

892 Thin section observations indicate that the shortening was accommodated by

893 localized dissolution between grains, and extension, by the precipitation of directed

894 overgrowths. Grain interiors are generally undeformed. The fiber overgrowths

895 mantle the grains in the $X$ direction and are unidirectionally aligned with that

896 direction. From these observations we conclude that the initial dimensions of the

897 grains are preserved in the $X$ direction. This conclusion holds for many pressure-

898 solved sandstones (Feehan and Brandon, 1999; Ring and Brandon, 1999; Ring et al.,

899 2001; Rahl et al., 2011).

900

901 Some care is needed to account for this heterogenous intergranular deformation.

902 We find it useful to identify how these different components of the deformation 
903 affect the bulk principal stretches. The change in grain shape, as caused by

904 dissolution, is represented by principal stretches $S_{g X}, S_{g Y}$, and $S_{g z}$. The contribution of

905 fiber overgrowth is represented by principal stretches $S_{f x}, S_{f y}$, and $S_{f z}$. The principal

906 stretches for the bulk deformation are given by $S_{X}=S_{g X} S_{f X}, S_{Y}=S_{g Y} S_{f Y}$, and $S_{Z}=S_{g Z} S_{f Z}$.

907

908 Considering that the grains have uniform interiors that compose a large fraction of

909 the image, our interpretation is that the ACF method provides estimates of the

910 change in grain dimensions (Table 2). The thin section evidence indicates that the

911 grain dimensions remain unchanged in the $X$ direction, so $S_{g X}=1$. The other

912 principal strains for the grain shape are $S_{g Y}=0.97$ and $S_{g X}=0.52$. The volume

913 stretch for the grains is $S_{g V}=S_{g X} S_{g Y} S_{g Z}$, which for this sample is $S_{g V} \approx 0.51$, or about

$91450 \%$ loss of grain volume.

915

916 For comparison, we also used the PDS method to measure grain stretches (Table 2).

917 PDS measurements are based on microscopy measurements made in 2D thin

918 sections, but they are corrected to account for stereological effects associated with

919 2D sections through 3D volumes (Rahl et al., 2011). The PDS method indicates $S_{g V} \approx$

920 0.76. The ACF method has the advantage that it provides a full estimate in $3 \mathrm{D}$, and is

921 also more precise than the PDS method.

922

923 We consider now the bulk deformation of this sample $S_{X}, S_{Y}$ and $S_{Z}$. Modal

924 measurements indicate that fiber overgrowth composes 15 percent of the sample.

925 For unidirectional fibers, the fiber-related stretch in the $X$ direction is $S_{f X}=1 /(1-m)$, 
926 where $m$ is the modal fraction of overgrowth (Feehan and Brandon, 1999). The bulk

927 strain in the $Y$ and $Z$ directions is limited to dissolution of the grain boundaries, so

$928 S_{f Y}=S_{f Z}=1$. Using the ACF results and the equations above for fiber and grain

929 strains, we estimate that the bulk strain in this sample caused by pressure solution

930 is $S_{X}=1.17, S_{Y}=0.97$, and $S_{Z}=0.52$, with a volume stretch of $S_{V}=0.59$. In other

931 words, the pressure-solution deformation generated a net $41 \%$ volume loss from

932 the bulk sample. Note that this analysis does not account for deformation associated

933 with compaction of the sandstone, as selvage and overgrowth fabrics indicate that

934 the sandstone had little porosity when pressure solution started (see Feehan and

935 Brandon, 1999 for further discussion of this issue).

936

937 The grain size is estimated by fitting the two-component model in (5) to the

938 estimated isotropic ACF. The grain size prior to deformation can be estimated by

939 scaling the lags so there is no strain in the $X$ direction. The estimated average grain

940 radius for this sample is $149 \mu m$ (Table 2).

941

$942 \quad 7.52$ D Image of Deformed Mg-Zr Alloy

943 Images and tomograms may also contain features that are unrelated to the

944 deformation of the sample, so care is required for proper interpretation of results.

945 We discuss here an example that illustrates this point.

946

947 Figure 15 here.

948 
949 Figure 15a shows a hydrided Mg-Zr alloy with minor Zr grains (Harris, 1973). Such

950 materials are commonly used in nuclear reactors due to their resistance to creep at

951 high temperatures (e.g., Wadsworth et al., 2001). The original fabric was formed by

952 inducing growth of $\mathrm{Zr}$ precipitates within the metal, and then passing the alloy

953 through rollers, which produces a flattening fabric. Later, the alloy was subjected to

954 a uniaxial extension, with $X$ oriented in the image vertical. The important point is

955 that the laminated fabric predates the extensional fabric. The alloy accommodated

956 about half of the extension by dilational "veins" or overgrowths, and the other half

957 by microfaulting of the rolled fabric. The overgrowth regions are called "denuded

958 zones" because they lack the $\mathrm{Zr}$ precipitates that were in the original alloy. The

959 fabric at present appears granular, but the granular structure is actually defined by

960 microfaults.

961

962 The modal abundance of the denuded zones is 4.6 percent (Harris, 1973), which

963 accommodated $4.6 \mathrm{cNp}$ of extension in the vertical. Harris (1973) suggested the

964 denuded zones formed by mass transfer associated with Nabarro-Herring creep

965 (see Xu et al., 2015, for an alternative interpretation), which implies that the

966 "grains" have internal deformation as well. The ACF method correctly estimated the

967 principal strain directions, but failed to correctly estimate the principal strains

968 (Table 1). This result is not surprising given that the laminar fabric has nothing to

969 do with the extensional deformation. One might speculate that the estimated

970 principal strains are representative of the full deformational history of the alloy,

971 including rolling and stretching, but we have no way to test that idea. 
973 Inspection of Fig. 15b shows that the deformed ACF field has a complicated

974 rectilinear pattern, quite different from the simple ellipsoidal pattern seen in the

975 previous examples. The ACF is clearly dominated by the $\mathrm{Zr}$ precipitates, which have

976 a large contrast with the other features in the image. One can see that the ACF is not

977 elliptical, which is an indication that the image is not suitable for strain estimation

978 using the ACF method.

979

980 Figure 15 here.

981

982 An alternative approach is to outline the "grains" and replace the laminated fabric 983 inside each grain with a uniform and unique grayscale value (Fig. 16). The strain

984 estimates for this example (Table 3) indicates that the "grains" are slightly elongate 985 in the vertical, but the method overestimated the principal strains. The reason that 986 this example fails to give useful strain estimates is that the "grains" are not passive 987 markers, but instead were produced by microfaulting. The ACF analysis could be 988 useful, in this case, for estimating the aspect ratio of fault-bounded domains, but it 989 cannot be expected to provide any information about strain.

990

991 Figure 16 here.

992

993 Table 3 here.

994 


\section{8. Discussion}

996 In this paper, we started with schematic examples that help illustrate how the ACF

997 might be used to estimate anisotropy and strain. This conceptual framework builds

998 on the pioneering work of Panozzo Heilbronner (1992). Our main contributions are

999 the introduction of a least-squares method for estimating anisotropy and strain

1000 from real images and tomograms, and a discussion of how different fabric features

1001 influence the ACF estimates. The examples in Tables 1-2 provide a useful test of the

1002 ACF method, and illustrate some complex cases where the method might fail or

1003 special care is required to get useful results.

1004

1005 We finish here with a discussion of some general issues. The first concerns the

1006 interpretation of the least-squares uncertainties. The next are three issues for future

1007 development of the ACF method.

1008

\section{$1009 \quad$ 8.1 Estimated Least-squares Uncertainties}

1010 Many readers may be skeptical of the small estimated uncertainties reported for our

1011 ACF estimates (Tables 1-2). What is important to remember is that the reported

1012 uncertainties are only representative of the uncertainty associated with the least-

1013 squares estimate. The least-squares method not only estimates a best-fit model, but

1014 also the residuals, which are the estimated errors in the data relative to that model.

1015 The uncertainties in the parameters are simply a function of how the variation in the

1016 residuals translates into variation in the estimated values of the parameters. 
1018 There may be other sources of error beyond those represented by the residuals. For 1019 example, the model may be incorrectly specified. The best check for a well-fit model

1020 is by inspection of the results, such as the deformed ACF, the undeformed ACF, and

1021 the residuals relative to the estimated isotropic lag. The $R^{2}$ and Durbin-Watson

1022 statistics provide additional diagnostic clues.

1023

1024 Usually, it is obvious when the model function fails. The Mg-Zr alloy provides a

1025 useful example, in that the contours of the deformed ACF are far from elliptical.

1026 Thus, we should know, long before applying the ACF method, that the method will

1027 probably not work. Yes, we have used the ACF method to calculate strain estimates

1028 (Table 1), but those estimates and their uncertainties are not useful estimates of the 1029 strain experienced by the alloy.

1030

1031 It might also be thought that heterogeneous strain at the grain scale may also

1032 invalidate the ACF results. The pressure-solved examples illustrate a common type

1033 of heterogeneous deformation, where the deformation is accommodated by

1034 dissolution and precipitation in different positions along grain boundaries.

1035 However, we have shown that the method seems to work well for estimating grain

1036 strain in the pressure solution examples, because the grains are the dominant

1037 features of the fabric.

1038

$1039 \quad 8.2$ Symmetry of the ACF 
1040 Our strain estimates have orthorhombic symmetry, because the model function is

1041 based on a strain tensor that cannot represent symmetry lower than orthorhombic.

1042 The ACF, however, can have lower than orthorhombic symmetry, requiring only an

1043 inversion center (Priestley, 1981; Panozzo Heilbronner, 1992). Therefore, we

1044 anticipate that the ACF method may be useful for studying low symmetry fabrics,

1045 such as those found in rocks from ductile shear zones. For example, the ellipticity of

1046 porphyroblasts may be measurably different from deformed neoblasts or micas in

1047 the surrounding matrix. Panozzo Heilbronner (1992) provided examples of ACFs

1048 with lower than orthorhombic symmetry, and some suggestions for interpreting

1049 these fabrics, such as the sense of shearing.

1050

$1051 \quad 8.3$ ACF Sensitivity

1052 In our opinion, the most important next step for the ACF method is to characterize

1053 the sensitivity of the ACF for different fabric features. The intensity provides a clue

1054 as to what features will dominate, but as Fig. 6 demonstrates, the influence of fabric

1055 features at any particular ACF lag value is also dependent on the feature size and

1056 spacing. If the ellipses in Fig. 6 had similar intensity values, the ACF would find

1057 parameters that are some average of the anisotropy of the two elliptical phases. A

1058 method of adjusting intensity values in the image to focus the ACF estimates of

1059 strain on different fabric elements would be useful. For the Mg-Zr alloy (Figs. 15 and

1060 16), segmenting the fault-bounded domains focused the ACF estimate of anisotropy

1061 on the fault-bounded domains, rather than the foliated Zr streaks. 
1063 For 3D objects, manual segmentation of each slice is labor intensive, and

1064 computerized segmentation methods (e.g., Ketcham, 2005b) may be especially

1065 useful. In many images, however, there may be too much overlap in the intensity

1066 values of different fabric features to allow for precise segmentation. We found this

1067 to be the case for the pressure-solved sandstone (Fig. 13). These cases require a

1068 continuous adjustment to the intensity values to emphasize different features. One

1069 approach would be to manually adjust the intensity values in Photoshop using the

1070 gamma-correction tool. The same correction can be automated and applied to each

1071 "slice" in a stack of images that makeup a tomogram. Alternatively, a statistical

1072 approach can also be used to continuously rescale the intensity values (Eisemann et 1073 al., 2011).

1074

1075 8.4. Dynamic Range and Neighbor Correlation

1076 An additional objective is to allow for estimation of anisotropy as a function of lag.

1077 At present, the X-ray tomogram images of the pressure-solved sandstone examples

1078 have excellent resolution for estimating anisotropy associated with self-correlation,

1079 but they lack the sample size and dynamic range to estimate anisotropy associated

1080 with neighbor-correlation.

1081

1082 To explain, let's consider first the requirements for minimum resolution. In our

1083 experience, the raw image should have a voxel size smaller than about 2.5 percent of

1084 the grain diameter. This value allows for " $2 \times 2$ binning", as needed to remove

1085 aliasing errors (Rivers et al., 2010). As a result, the actual operational image should 
1086 have a voxel size smaller than $5 \%$ of the grain diameter. For comparison, the 1087 tomogram for our pressure-solved sample has voxel sizes of $7.54 \mu \mathrm{m}$, which works 1088 out to $\sim 3 \%$ of the grain diameter $\left(2 R_{S} \approx 220 \mu \mathrm{m}\right)$. One might consider smaller 1089 voxels if there are fine features, such as overgrowths or selvages, that need to be 1090 identified for modal analysis or removed by image processing. Accurate model 1091 analysis commonly will require a voxel resolution of 1 to $5 \mu \mathrm{m}$.

1093 What we are lacking at present is sufficient dynamic range, which would allow 1094 images of larger samples while maintaining the resolution. For example, our 1095 tomogram for the pressure-solved sandstone has an average of $\sim 6$ grain diameters 1096 across each dimension. This size is sufficient for determining self-correlation, but it 1097 is inadequate to fully resolve neighbor-correlation. For example, Crespi (1986) 1098 estimated that for the Fry method in 2D, measurements of about 500 to 1000 grains 1099 are needed to get a successful solution. This works out to an image that has an 1100 average of 22-32 grains across each dimension. Thus, the pressure-solved 1101 sandstone, which has an average grain diameter of $\sim 220 \mu \mathrm{m}$, would require 1102 tomograms that are 5 to $8 \mathrm{~mm}$ in each dimension. The raw image, after binning,

1103 would have to be 880 to 1200 voxels per dimension. For comparison, each working 1104 dimension of our cropped tomograms is about 400 to 500 voxels.

1106 X-ray tomography imaging can be divided into two types of setups: commercially 1107 available desktop scanners and synchrotrons. Commercial scanners use X-ray tubes, 1108 in which a target, such as tungsten, is impacted by an electron beam to generate X- 
1109 rays. For synchrotrons, electrons traveling at velocities near the speed of light are

1110 accelerated in storage rings using bending magnets, undulators, and wigglers. The

1111 electrons produce X-rays as they circle through the storage ring, and those X-rays

1112 are extracted at fixed points around the ring. The main difference between

1113 commercial and synchrotron scanners is that the synchrotron has a much larger

1114 photon flux spread over a larger range of X-ray energy (Fusseis et al., 2014). As a

1115 result, the X-ray beam can be filtered to specific energy bands while maintaining a

1116 large enough photon flux for tomography. Selecting a specific energy band is useful

1117 for avoiding beam-hardening artifacts, or optimizing attenuation contrast. In

1118 addition, a large photon flux means that the tomogram can be acquired quickly, on

1119 the order of 10 minutes at present, and with low image noise. In contrast, the lower

1120 photon flux for commercial systems means acquisition times on the order of 1.5 to 4

1121 hours for similar sample sizes.

1122

1123 The voxel resolution and sample size requirements also differ between commercial

1124 and synchrotron systems. The Argonne APS Beamline 13-BM D can image a cylinder

1125 with a diameter less than $50 \mathrm{~mm}$ and a height less than $4 \mathrm{~mm}$. The CCD in use during

1126 our measurements provided 650 pixels across the radial dimension after binning for

1127 aliasing. If we consider an $8 \mathrm{~mm}$ diameter cylinder, the voxel resolution would be

1128 about $12 \mu \mathrm{m}$ (see Gualda and Rivers, 2006, for further details). Commercial systems

1129 are more flexible with sample size and can provide voxel sizes down to the micron

1130 scale for small samples, but as noted above the acquisition times are much longer.

1131 
1132 The small sample size creates another problem, in that it is challenging to keep the

1133 sample oriented relative to its geographic orientation. Given our experiences, we

1134 recommend that samples be prepared as small cores, on the order of $8 \mathrm{~mm}$ in

1135 diameter, which is about the diameter of a pencil, and several $\mathrm{cm}$ in length. The

1136 azimuthal direction of the core could be marked with a scribe. Using this approach,

1137 the orientation of the core can then be directly acquired from the tomogram. For

1138 comparison, note that a rock chip for a standard thin-section billet is about $26 \mathrm{~mm} \mathrm{x}$

$113946 \mathrm{~mm} \times 10 \mathrm{~mm}$.

1140

\section{9. Conclusions}

1142 We have outlined the utility of the ACF method for estimating anisotropy and

1143 deformation. Our analyses here have emphasized thin-section images and X-ray

1144 tomograms, but clearly the ACF method can be applied to other kinds of field

1145 variables, such as composition and crystal orientation. In comparison to other

1146 methods of strain estimation, the ACF method allows for more precise

1147 representations of objects, and does not require fitting ellipses to objects or

1148 abstractions of grain boundaries. The method also works equally well for both 2D

1149 and 3D images.

1150

1151 The method works best for images or tomograms where grains have a uniform

1152 internal intensity but a strong contrast with the intensity of other grains and matrix.

1153 For example, a tomogram of a granite or a polymictic sandstone should work well,

1154 but a tomogram of a monomineralic rock, such as a limestone, dunite, or quartzite, 
1155 would likely give poor results unless there are significant variations in attenuation

1156 between grains. It is also a challenge to work with grains that have a complicated

1157 internal structure, as illustrated by the examples of the ironstone oolite and the Mg-

$1158 \mathrm{Zr}$ alloy. There are ways to treat these cases, such as manually segmenting grains, as

1159 was done for the metal example. In this regard, computerized segmentation

1160 methods (Ketcham; 2005b) may prove useful, especially for 3D tomograms. Right

1161 now, our analysis is weighted towards the self-correlation component of the ACF,

1162 and thus is most sensitive to particle shapes. Further work is needed to develop

1163 methods that would be able to provide separate estimates of the anisotropies

1164 associated with self-correlation and neighbor-correlation. The method could also be

1165 generalized to give a continuous estimate of anisotropy as a function of lag.

1166

\section{Acknowledgements}

1168 We thank Mark Rivers for his help in imaging sample 92810-3, Jean Crespi for

1169 providing the photomicrograph of the ooids from the Jurassic Stump formation, and

1170 John Wheeler for introducing us to the Mg-Zr alloy example. We thank Kieran

1171 Mulchrone, an anonymous reviewer, and the Editor William Dunne for their

1172 constructive and helpful comments. C.J.T. acknowledges support from an NSF

1173 Graduate Research Fellowship under grant DGE-1122492 and from the Stephen E.

1174 Laubach Award in Structural Diagenesis. This research used resources of the

1175 Advanced Photon Source, a U.S. Department of Energy (DOE) Office of Science User

1176 Facility operated for the DOE Office of Science by Argonne National Laboratory

1177 under Contract No. DE-AC02-06CH11357. 


\section{References}

1180 Alexander, T., 1997. Is AGN Variability Correlated with Other AGN Properties?-

1181 ZDCF Analysis of Small Samples of Sparse Light Curves. In: Maoz, D. Sternberg, A.

1182 Leibowitz E.M. (Eds), Astronomical Time Series. Springer, Netherlands, 163-166.

1184 Andrä, H., Combaret, N., Dvorkin, J., Glatt, E., Han, J., Kabel, M., Keehm, Y., Krzikalla,

1185 F., Lee, M., Madonna, C., Marsh, M., Mukerji, T., Saenger, E.H., Sain, R., Saxena, N.,

1186 Ricker, S., Wiegmann, A., Zhan, X., 2013. Digital rock physics benchmarks-Part I:

1187 Imaging and segmentation. Computers \& Geosciences, 50, 25-32.

1188 doi:10.1016/j.cageo.2012.09.005

1189

1190 Baker, D. R., Mancini, L., Polacci, M., Higgins, M. D., Gualda, G. A. R., Hill, R. J., \& Rivers,

1191 M. L., 2012. An introduction to the application of X-ray microtomography to the

1192 three-dimensional study of igneous rocks. Lithos, 148, 262-276. doi:

1193 10.1016/j.lithos.2012.06.008

1194

1195 Baveye, P.C., Laba, M., Otten, W., Bouckaert, L., Sterpaio, P.D., Gowami, R.R., Grinev,

1196 D., Houston, A., Hu, Y., Liu, J., Mooney, S., Pajor, R., Sleutel, S., Tarquis, A., Wang, W.,

1197 Wei, Q., Sezgin, M., 2010. Observer-dependent variability of the thresholding step in

1198 the quantitative analysis of soil images and X-ray microtomography data, Geoderma, 1199 157, 51-63, doi:10.1016/j.geoderma.2010.03.015.

1201 Boone, M., Dewanckele, J., Boone, M., Cnudde, V., Silversmit, G., Van Ranst, E., Jacobs,

1202 P., Vincze L., Van Hoorebeke, L., 2011. Three-dimensional phase separation and

1203 identification in granite. Geosphere, 7(1), 79-86. doi: 10.1130/GES00562.1

1204

1205 Berger, M.J., Hubbell, J.H., Seltzer, S.M., Chang, J., Coursey, J.S., Sukumar, R., 1206 Zucker, D.S., and Olsen, K., 2010. XCOM: Photon Cross Section Database

1207 (version 1.5). [Online] Available: http://physics.nist.gov/xcom. National Institute of 
1208 Standards and Technology, Gaithersburg, MD.

1209

1210 Bevington, P. R., \& Robinson, D. K. (2003). Data reduction and error analysis.

1211 McGraw-Hill, New York.

1212

1213 Box, G. E., \& Cox, D. R., 1964. An analysis of transformations. Journal of the Royal

1214 Statistical Society. Series B (Methodological), 211-252.

1215

1216 Brabant, L., Vlassenbroeck, J., De Witte, Y., Cnudde, V., Boone, M. N., Dewanckele, J.,

1217 Van Hoorebeke, L., 2011. Three-dimensional analysis of high-resolution X-ray

1218 computed tomography data with Morpho+. Microscopy and Microanalysis, 17(02),

1219 252-263. doi:10.1017/S1431927610094389

1220

1221 Brandon, M. T., 1995. Analysis of geologic strain data in strain-magnitude space.

1222 Journal of Structural Geology, 17(10), 1375-1385. doi: 10.1016/0191-

1223 8141(95)00032-9

1224

1225 Brandon, M. T., Roden-Tice, M. K., Garver, J. I., 1998. Late Cenozoic exhumation of the

1226 Cascadia accretionary wedge in the Olympic Mountains, northwest Washington

1227 State. Geological Society of America Bulletin, 110(8), 985-1009. doi: 10.1130/0016-

1228 7606(1998)110<0985:LCEOTC>2.3.CO;2

1229

1230 Broersen, P. M., 2006. Automatic autocorrelation and spectral analysis. London:

1231 Springer.

1232

1233 Brun, F., Mancini, L., Kasae, P., Favretto, S., Dreossi, D., \& Tromba, G., 2010. Pore3D: a

1234 software library for quantitative analysis of porous media. Nuclear Instruments and

1235 Methods in Physics Research Section A: Accelerators, Spectrometers, Detectors and

1236 Associated Equipment, 615(3), 326-332. doi: 10.1016/j.nima.2010.02.063

1237 
1238 Buscombe, D., Masselink, G., 2009. Grain-size information from the statistical

1239 properties of digital images of sediment. Sedimentology, 56: 421-438.

1240 doi:10.1111/j.1365-3091.2008.00977.x

1241

1242 Carlson, W. D., 2006. Three-dimensional imaging of earth and planetary materials.

1243 Earth and Planetary Science Letters, 249(3), 133-147.

1244 doi:10.1016/j.epsl.2006.06.020

1245

1246 Chantler, C.T., Olsen, K., Dragoset, R.A., Chang, J., Kishore, A.R., Kotochigova, S.A., 1247 Zucker, D.S. (2005), X-Ray Form Factor, Attenuation and Scattering Tables (version

1248 2.1). [Online] Available: http://physics.nist.gov/ffast. National Institute of Standards 1249 and Technology, Gaithersburg, MD.

1250

1251 Cnudde, V., Boone, M. N., 2013. High-resolution X-ray computed tomography in 1252 geosciences: A review of the current technology and applications. Earth-Science 1253 Reviews, 123, 1-17. doi:10.1016/j.earscirev.2013.04.003

1254

1255 Crespi, J. M., 1986. Some guidelines for the practical application of Fry's method of

1256 strain analysis. Journal of Structural Geology, 8(7), 799-808. doi:10.1016/0191-

1257 8141(86)90026-X

1258

1259 Cule, D., Torquato, S., 1999. Generating random media from limited microstructural 1260 information via stochastic optimization. Journal of applied physics, 86(6), 34281261 3437. doi:10.1063/1.371225.

1262

1263 Debye, P., \& Bueche, A. M. (1949). Scattering by an inhomogeneous solid. Journal of 1264 Applied Physics, 20(6), 518-525.

1265

1266 Debye, P., Anderson Jr, H. R., Brumberger, H., 1957. Scattering by an inhomogeneous

1267 solid. II. The correlation function and its application. Journal of applied Physics, 1268 28(6), 679-683. doi:10.1063/1.1722830. 
1270 Denison, C., Carlson, W. D., Ketcham, R. A., 1997, Three-dimensional quantitative

1271 textural analysis of metamorphic rocks using high-resolution computed X-ray

1272 tomography: Part I. Methods and techniques. Journal of Metamorphic Geology,

1273 15: 29-44. doi: 10.1111/j.1525-1314.1997.00006.x.

1274

1275 Denison, C., Carlson, W. D., 1997. Three-dimensional quantitative textural analysis of

1276 metamorphic rocks using high-resolution computed X-ray tomography: Part II.

1277 Application to natural samples. Journal of Metamorphic Geology, 15: 45-57,

1278 doi:10.1111/j.1525-1314.1997.00007.x.

1279

1280 Donev, A., Torquato, S., Stillinger, F. H., 2005. Neighbor list collision-driven

1281 molecular dynamics simulation for nonspherical hard particles. I. Algorithmic

1282 details, Journal of Computational Physics, 202(2). 737-764.

1283 doi:10.1016/j.jcp.2004.08.014

1284

1285 Donev, A., Torquato, S., \& Stillinger, F. H. (2005). Neighbor list collision-driven

1286 molecular dynamics simulation for nonspherical hard particles.: II. applications to

1287 ellipses and ellipsoids. Journal of Computational Physics, 202(2), 765-793.

1288

1289 Donev, A., 2006. Jammed packings of hard particles, Ph.D. thesis. Princeton

1290 University.

1291

1292 Draper, N. R., Smith, H., 1998. Applied regression analysis (3rd ed.). Wiley-

1293 Interscience. New York. ISBN 0-471-17082-8

1294

1295 Ebel, D. S. and Rivers, M. L., 2007. Meteorite 3-D synchrotron microtomography:

1296 Methods and applications. Meteoritics \& Planetary Science, 42: 1627-1646.

1297 doi:10.1111/j.1945-5100.2007.tb00595.x

1298 
1299 Eisemann, M., Albuquerque, G., \& Magnor, M. (2011). Data driven color mapping. In 1300 Proceedings of EuroVA: International Workshop on Visual Analytics, Bergen, 1301 Norway.

1302

1303 Elangovan, P., Hezel, D. C., Howard, L., Armstrong, R., Abel, R. L., 2012. PhaseQuant: A 1304 tool for quantifying tomographic data sets of geological specimens. Computers \& 1305 Geosciences, 48, 323-329. doi:10.1016/j.cageo.2012.01.014 1306

1307 Efron, B., 1979. Bootstrap methods: another look at the jackknife. The Annals of 1308 Statistics, 7, 1-26.

1309

1310 Erslev, E. A., 1988. Normalized center-to-center strain analysis of packed aggregates. 1311 Journal of Structural Geology, 10(2), 201-209. doi: 10.1016/0191-8141(88)90117-4 1312

1313 Feehan, J. G., M. T. Brandon, 1999. Contribution of ductile flow to exhumation of low1314 temperature, high-pressure metamorphic rocks: San Juan-Cascade nappes, NW 1315 Washington State, J. Geophys. Res., 104(B5), 10883-10902, 1316 doi:10.1029/1998JB900054.

1318 Friese, K. I., Cichy, S. B., Wolter, F. E., Botcharnikov, R. E., 2013. Analysis of 1319 tomographic mineralogical data using YaDiV-Overview and practical case study. 1320 Computers \& Geosciences, 56, 92-103, doi: 10.1016/j.cageo.2013.01.021.

1321

1322 Fry, N., 1979. Random point distributions and strain measurement in rocks.

1323 Tectonophysics, 60(1), 89-105.

1324

1325 Fusseis, F., Xiao, X., Schrank, C., \& De Carlo, F., 2014. A brief guide to synchrotron

1326 radiation-based microtomography in (structural) geology and rock mechanics.

1327 Journal of Structural Geology, 65, 1-16.

1328 
1329 Gualda G.A.R., Rivers M., 2006. Quantitative 3D petrography using X-ray

1330 tomography: Application to Bishop Tuff pumice clasts. Journal of Volcanology and 1331 Geothermal Research 154: 48-62.

1332

1333 Gualda, G. A., Pamukcu, A. S., Claiborne, L. L., \& Rivers, M. L., 2010. Quantitative 3D

1334 petrography using X-ray tomography 3: Documenting accessory phases with

1335 differential absorption tomography. Geosphere, 6(6), 782-792. doi:

$1336 \quad 10.1130 /$ GES00568.1

1337

1338 Harris, J. E., 1973. The inhibition of diffusion creep by precipitates. Metal Science, 1339 7(1), 1-6. Doi: 10.1179/030634573790445514

1340

1341 Heilbronner, R., 2010. Mapping texture domains in quartzite microstructures.

1342 Journal of the Geological Society of India, 75(1), 160-170.

1343

1344 Heilbronner, R., Barrett, S., 2013. Image analysis in earth sciences: microstructures 1345 and textures of earth materials. Springer.

1346 Hencky, H., 1931. The Law of Elasticity for Isotropic and Quasi-Isotropic Substances

1347 by Finite Deformations. Journal of Rheology (1929-1932), 2(2), 169-176.

1348 doi:/10.1122/1.2116361.

1349

1350 Hext, G.R., 1963. The estimation of second-order tensors, with related tests and

1351 designs. Biometrika, 50(3-4), 353-373, doi:10.1093/biomet/50.3-4.353.

1352

1353 Higham, N. J., 2008. Functions of matrices: theory and computation. SIAM,

1354 Philadelphia.

1355

1356 Hubbell, J.H. Seltzer, S.M., 2004. Tables of X-Ray Mass Attenuation Coefficients and

1357 Mass Energy-Absorption Coefficients (version 1.4). [Online] Available:

1358 http://physics.nist.gov/xaamdi. National Institute of Standards and Technology,

1359 Gaithersburg, MD. 
1361 Huddlestone-Holmes, C.R., Ketcham, R.A., 2005. Getting the inside story: using 1362 computed X-ray tomography to study inclusion trails in garnet porphyroblasts.

1363 American Mineralogist, November-December, v. 90, ea1-ea17,

1364 doi:10.2138/am.2005.1840

1365

1366 Hu, Q., Ley, M.R., Davis, J., Hana J.C., Frazier, R., Zhang, Y., 2014. 3D chemical

1367 segmentation of fly ash particles with X-ray computed tomography and electron

1368 probe microanalysis, Fuel, 116(15) 229-236, doi:10.1016/j.fuel.2013.07.037.

1369

1370 Iassonov, P., Gebrenegus, T., Tuller, M., 2009, Segmentation of X-ray computed

1371 tomography images of porous materials: A crucial step for characterization and

1372 quantitative analysis of pore structures, Water Resour. Res., 45, W09415,

1373 doi:10.1029/2009WR008087.

1374

1375 Iassonov, P., Tuller, M., 2010. Application of segmentation for correction of intensity

1376 bias in X-ray computed tomography images. Vadose Zone Journal, 9(1), 187-191.

1377 doi:10.2136/vzj2009.0042.

1378

1379 Jelínek, V., 1978. Statistical processing of anisotropy of magnetic susceptibility

1380 measured on groups of specimens. Studia geophysica et geodaetica, 22(1), 50-

1381 62.doi: 10.1007/BF01613632.

1382

1383 Jerram, D. A., Higgins, M. D., 2007. 3D analysis of rock textures: quantifying igneous

1384 microstructures. Elements, 3(4), 239-245, doi:10.2113/gselements.3.4.239.

1385

1386 Kansal, A. R., Torquato, S., Stillinger, F. H., 2002. Computer generation of dense

1387 polydisperse sphere packings. The Journal of chemical physics, 117(18), 8212-8218.

1388 doi:10.1063/1.1511510.

1389 
1390 Ketcham, R. A., 2005a. Three-dimensional grain fabric measurements using high1391 resolution X-ray computed tomography. Journal of Structural Geology, 27(7), 121713921228.

1393

1394 Ketcham, R. A., 2005b. Computational methods for quantitative analysis of three-

1395 dimensional features in geological specimens. Geosphere, 1(1), 32-41.

1396 doi:10.1130/GES00001.1

1397

1398 Ketcham, R.A., Carlson, W.D., 2001. Acquisition, optimization and interpretation of

1399 X-ray computed tomographic imagery: applications to the geosciences. Computers \& 1400 Geosciences 27.4, 381-400. 10.1016/S0098-3004(00)00116-3.

1401

1402 Ketcham, R. A. and Ryan, T. M., 2004. Quantification and visualization of anisotropy

1403 in trabecular bone. Journal of Microscopy, 213: 158-171. doi:10.1111/j.1365-

1404 2818.2004.01277.x

1405

1406 Lado, F., Torquato, S., 1990. Two-point probability function for distributions of

1407 oriented hard ellipsoids. The Journal of chemical physics, 93(8), 5912-5917.

1408 doi:10.1063/1.459501

1409

1410 Lindquist, W. B., Venkatarangan, A., Dunsmuir, J., Wong, T., 2000. Pore and throat

1411 size distributions measured from synchrotron X-ray tomographic images of

1412 Fontainebleau sandstones, J. Geophys. Res., 105(B9), 21509-21527,

1413 doi:10.1029/2000JB900208.

1414

1415 Liu, J., Regenauer-Lieb, K., Hines, C., Liu, K., Gaede, O., Squelch, A., 2009. Improved

1416 estimates of percolation and anisotropic permeability from 3-D X-ray

1417 microtomography using stochastic analyses and visualization, Geochem. Geophys.

1418 Geosyst., 10, Q05010, doi:10.1029/2008GC002358 
1420 Luding, S., 2004. Molecular Dynamics Simulations of Granular Materials. In:

1421 Hinrichsen, H., Wolf D.E. (Eds.), The Physics of Granular Media. Wiley-VCH Verlag

1422 GmbH \& Co. KGaA, Weinheim, FRG, doi: 10.1002/352760362X.ch13

1423

1424 Lubachevsky, B., Stillinger, F.H., 1990. Geometric properties of random disk

1425 packings. Journal of Statistical Physics, 60(5-6), 561-583.

1426

1427 Maätzler, C., 2002. Relation between grain-size and correlation length of snow.

1428 Journal of Glaciology, 48(162), 461-466. doi: 10.3189/172756502781831287

1429

1430 Maire, E., Withers, P.J., 2014. Quantitative X-ray tomography. International Materials

1431 Reviews, 59(1), 1-43.

1432

1433 Mills, I. M. (1995). Unity as a Unit. Metrologia, 31(6), 537. doi:10.1088/0026-

$14341394 / 31 / 6 / 013$

1435

1436 Moler, C. B., 2008. Numerical computing with MATLAB. SIAM. Philadelphia. ISBN

1437 978-0-89871-660-3

1438

1439 Mookerjee, M., Peek, S., 2014. Evaluating the effectiveness of Flinn's k-value versus

1440 Lode's ratio. Journal of Structural Geology. doi: 10.1016/j.jsg.2014.08.008

1441

1442 O'Connor, A., Mulchrone, K. F., \& Meere, P. A., 2009. WinDICOM: A program for

1443 determining inclusion shape and orientation, Computers \& Geosciences 35(6):

1444 1358-1368. doi:10.1016/j.cageo.2008.08.015

1445

1446 Owens, W.H., 2000. Statistical applications to second-rank tensors in magnetic

1447 fabric analysis, Geophys. J. Int 142 (2): 527-538 doi:10.1046/j.1365-

1448 246x.2000.00174.x

1449 
1450 Panozzo Heilbronner, R., 1992. The autocorrelation function: an image processing 1451 tool for fabric analysis. Tectonophysics, 212(3), 351-370.

1452

1453 Press, W.H., Teukolsky, S.A., Vetterling, W.T., Flannery, B.P., 2007. Numerical recipes

1454 3rd edition: The art of scientific computing. Cambridge University Press, Cambridge.

1455

1456 Priestley, M.B., 1981. Spectral Analysis and Time Series. Academic Press, San Diego. 1457

1458 Rahl, J. M., Brandon, M. T., Deckert, H., Ring, U., \& Mortimer, N., 2011. Tectonic

1459 significance of ductile deformation in low-grade sandstones in the mesozoic Otago

1460 subduction wedge, New Zealand. American Journal of Science, 311(1), 27-62. doi:

$1461 \quad 10.2475 / 01.2011 .02$

1462

1463 Ramsay, J. G. (1967). Folding and fracturing of rocks. McGraw-Hill Companies. 1464

1465 Ramsay, J. G., Huber, M. I., 1983. The techniques of modern structural geology, Vol.

1466 1., Academic Press, London.

1467

1468 Reeder, R. J., Lanzirotti, A., 2006. Accessing user facilities and making your research

1469 experience successful. Elements, 2(1), 31-35. doi:10.2113/gselements.2.1.31

1470

1471 Ring, U., Brandon, M. T., 1999. Ductile deformation and mass loss in the Franciscan

1472 subduction complex: implications for exhumation processes in accretionary wedges.

1473 Special Publication-Geological Society of London, 154, 55-86.

1474 doi:10.1144/GSL.SP.1999.154.01.03

1475

1476 Ring, U., Brandon, M.T., Ramthun, A., 2001. Solution-mass-transfer deformation

1477 adjacent to the Glarus Thrust, with implications for the tectonic evolution of the

1478 Alpine wedge in eastern Switzerland. Journal of Structural Geology 23, 1491-1505.

1479 doi:10.1016/S0191-8141(01)00015-3.

1480 
1481 Rivers, M. L., Sutton, S. R., Eng, P. J., 1999. Geoscience applications of x-ray computed 1482 microtomography. In SPIE's International Symposium on Optical Science,

1483 Engineering, and Instrumentation International Society for Optics and Photonics. $1484 \quad 78-86$.

1485

1486 Rivers, M. L., Citron, D. T., Wang, Y., 2010. Recent developments in computed 1487 tomography at GSECARS. In SPIE Optical Engineering+ Applications, International 1488 Society for Optics and Photonics, 780409-780409.

1490 Ryan, T.M., Ketcham, R.A., 2002. The three-dimensional structure of trabecular bone 1491 in the femoral head of strepsirrhine primates. Journal of human evolution, 43(1), 11492 26, doi: 10.1006/jhev.2002.0552.

1493

1494 Salavon, J., 1998. The Class of 1988 \& The Class of 1967. Retrieved from

1495 http://www.salavon.com

1496

1497 Shepp, L. A., Logan, B. F., 1974. The Fourier reconstruction of a head section. Nuclear 1498 Science, IEEE Transactions on, 21(3), 21-43. doi: 10.1109/TNS.1974.6499235.

1500 Shimamoto, T., Ikeda, Y., 1976. A simple algebraic method for strain estimation from

1501 deformed ellipsoidal objects. 1. Basic theory. Tectonophysics, 36(4), 315-337, doi:

1502 10.1016/0040-1951(76)90107-4.

1503

1504 Skoge, M., Donev, A., Stillinger, F. H., Torquato, S., 2006. Packing hyperspheres in

1505 high-dimensional Euclidean spaces. Physical Review E, 74(4), 041127.

1506 doi:10.1103/PhysRevE.74.041127

1507

1508 Spencer, A. J. M., 1980. Continuum mechanics. Dover Publications, Mineola, N.Y. 1509

1510 Sutton, S. R., Bertsch, P. M., Newville, M., Rivers, M., Lanzirotti, A., Eng, P., 2002.

1511 Microfluorescence and microtomography analyses of heterogeneous earth and 
1512 environmental materials. Reviews in Mineralogy and Geochemistry, 49(1), 429-483.

1513 doi:10.2138/rmg.2002.49.8.

1514

1515 Svitelman, V., Dinariev, O., 2013. Geostatistical approach to the anisotropy analysis

1516 of 3D rock microtomographic models. Computers \& Geosciences, 57, 116-123.

1517 doi:10.1016/j.cageo.2013.04.007

1518 Tabor, R. W., \& Cady, W. M. (1978). The structure of the Olympic Mountains,

1519 Washington: Analysis of a subduction zone (Vol. 1033). US Govt. Print. Off..

1520

1521 Torquato, S., 2002a. Random heterogeneous materials: microstructure and

1522 macroscopic properties, Springer, New York.

1523

1524 Torquato, S., Lado, F., 1991. Trapping constant, thermal conductivity, and the

1525 microstructure of suspensions of oriented spheroids. The Journal of Chemical

1526 Physics, 94(6), 4453-4462. doi:10.1063/1.460635

1527

1528 Torquato, S., Stell, G., 1982. Microstructure of two-phase random media. I. The n-

1529 point probability functions. The Journal of Chemical Physics, 77(4), 2071-2077.

1530 doi:10.1063/1.444011

1531

1532 Torquato, S., Stell, G., 1983. Microstructure of two-phase random media. III. The n-

1533 point matrix probability functions for fully penetrable spheres. The Journal of

1534 Chemical Physics, 79(3), 1505-1510. doi:10.1063/1.445941

1535

1536 Torquato, S., Stell, G., 1985. Microstructure of two-phase random media. V. The n-

1537 point matrix probability functions for impenetrable spheres. The Journal of chemical

1538 physics, 82(2), 980-987.

1539

1540 Wadsworth, J., Ruano, O. A., Sherby, O. D., 2002. Denuded zones, diffusional creep, 1541 and grain boundary sliding. Metallurgical and Materials Transactions A, 33(2), 219-

1542 229, doi:10.1007/s11661-002-0084-7. 
1543

1544 Wright, T. O., Platt, L. B., 1982. Pressure dissolution and cleavage in the Martinsburg 1545 Shale. American Journal of Science, 282(2), 122-135, doi: 10.2475/ajs.282.2.122.

1546

1547 Xu, W.F., Zhang Y., Peng, L.M., Ding, W.J., Nie, J.F., 2015. Formation of denuded zones

1548 in crept Mg-2.5Gd-0.1Zr alloy, Acta Materialia. 84(1), 317-329

1549 doi:10.1016/j.actamat.2014.10.046.

1550

1551 


\section{Figure Captions}

1553 Figure 1. X-ray tomography image of an $X-Z$ section through a sandstone deformed

1554 by pressure solution (sample 92810-3 from the Olympic subduction complex in NW

1555 Washington State). A deformation fabric is indicated by detrital grains (dark gray),

1556 which are separated by anastomosing cleavage surfaces (long, thin light-gray

1557 features, approximately parallel to the $X$ direction) and by directed overgrowths

1558 (thicker light-gray features, oriented in the $X$ direction). The cleavages have

1559 accommodated shortening in the $Z$ direction, and the overgrowths, extension in the

$1560 X$ direction.

1561

1562 Figure 2: X-ray attenuation coefficients for various geologic materials (Chantler et

1563 al., 2005), as normalized by the coefficient for quartz. The gray regions show a

1564 "factor of two" range for an attenuation coefficient relative to that for quartz.

1565 Minerals that lie outside that range will be easily resolved relative to quartz in an X-

1566 ray tomogram.

1567

1568 Figure 3: Correlated structure as illustrated using averaging. (a) An average of

1569 yearbook pictures for women in the 1967 graduation class at Fort Worth High

1570 School (Salavon, 1998). (b) An average of $200 X-Z$ sections from an X-ray tomogram

1571 of deformed sandstone, representing an out-of-plane thickness of $392 \mu \mathrm{m}$ (sample

$157292810-3$ from the Olympic subduction complex).

1573 
1574 Figure 4. The autocorrelation function for three examples: (a) a single circle, (b) a

1575 square lattice of circles, and (c) an anticlustered distribution of circles. (d) The

1576 autocorrelation function $\rho(r)$ for these examples is related to the change in the

1577 overlapping area of the circles, which is set to 1 when the lag $r=0$, and decreases as

1578 the lag increases to $R$, the radius of the circles.

1579

1580 Figure 5. The Ramsay and Fry effects in the ACF. (a, b) At short lags, where

$1581 r / R<1$, the ACF represents the correlation of features with themselves, and thus

1582 the ACF is sensitive to the anisotropy of features. The anisotropy in this range is

1583 similar to that measured by the Ramsay method, which is focused on the distortion

1584 of features. (c, d) At long lags, where $r / R>1$, the ACF is sensitive to the

1585 anisotropic distribution of features with respect to each other. This anisotropy is

1586 similar to that measured by the Fry method, which focuses on the distribution of

1587 nearest neighbors.

1588

1589 Figure 6. Two examples showing how the ACF is affected by the contrast in

1590 intensity between phases. The ACFs for these examples are shown as contours of $\rho$

1591 in terms of $x$ and $y$ components of the lag vector and a contour interval of 0.1. (a) For

1592 the first example, the gray ellipses are small and have little contrast with the

1593 surrounding white phase, so (b) the ACF contours everywhere mimic the anisotropy

1594 of the larger black ellipses. (c) For the second example, the black ellipses are small,

1595 but have high contrast with respect to the other two phases. (d) As a result, the ACF 
1596 contours have the same elliptical shape as the black phase at short lags $\left(r / R_{X} \sim 0.1\right)$,

1597 but take on the elliptical shape of the gray phase at long lags $\left(r / R_{X}>\sim 0.3\right)$.

1598

1599 Figure 7. Comparison of ACF model functions. (a) A 3D synthetic aggregate

1600 composed of non-overlapping identical spheres. (b) The ACF for the synthetic

1601 aggregate (gray line) and best-fit solutions using the exponential model (dash line),

1602 the spherical model (solid line), and the 2-component model (dash-dot line). The

1603 "half-height" lag is shown by $r^{*}$. (c) The best-fit 2-component model (solid line) and

1604 the self-correlation (dash-dot line) and neighbor-correlation (dash line)

1605 components for that fit.

1606

1607 Figure 8. The uncertainty for a best-fit principal direction is represented by an

1608 elliptical cone (gray line) that intersects the plane normal to the direction. The radii

1609 of the cone are given by $\max \alpha_{95}$ and $\min \alpha_{95}$, and the orientation in the plane is

1610 given by the twist. This example shows the elliptical cone for the $Y$ principal

1611 direction. Positive $X$ and $Z$ directions are shown for a right-handed coordinate

1612 system where $Y$ points out of the image.

1613

1614 Figure 9. Synthetic example (phantom) used to test the ACF method for estimating

1615 3D strain. (a) A 3D rendering of the phantom, which was constructed from identical

1616 non-overlapping spheres and then deformed homogenously. (b) Observed ACF in

1617 the deformed coordinate frame with lag normalized to the radius $R$ of the 
1618 undeformed spheres. (c) Isotropic ACF, determined by removing estimated strain.

1619 (d) Standardized residuals for the best-fit isotropic ACF as a function of lag.

1620

1621 Figure 10. Use of ACF method to estimate strain for (a) 2D photomicrograph of

1622 undeformed ooids from the Jurassic Stump formation in Wyoming (Crespi, 1986).

1623 See caption in Figure 8 for other details, and Table 1 for results.

1624

1625 Figure 11. Use of ACF method to estimate strain for (a) 2D photomicrograph of

1626 deformed ooids (figure 7.7 in Ramsay and Huber, 1983). See caption in Figure 8 for

1627 other details, and Table 1 for results.

1628

1629 Figure 12. The effect of internal particle structure on the ACF. (a) A test object

1630 composed of concentric rings with the same aspect ratio. (b) The ACF contours are

1631 not everywhere elliptical, but instead have "hot-dog" shapes at intermediate lags.

1632 The black line is $r^{*}$.

1633

1634 Figure 13. Tomogram for deformed sandstone (sample 92810-3 from the Olympic

1635 subduction complex). (a) 3D rendering showing principal strain axes sections as

1636 determined using the ACF method. (b-d) Slices through the tomogram along

1637 principal strain sections. See Table 2 for results. The black box in panel (b) outlines

1638 the region shown in Fig. 14.

1639 
1640 Figure 14. Pressure-solution fabric elements in $X-Z$ slice of sample 92810-3

1641 tomogram. Arrows indicate quartz/feldspar and lithic grains, fibrous overgrowths,

1642 and selvages. The section area is outline by the black box in Fig. 13b.

1643

1644 Figure 15. A cautionary tale for using the ACF method. (a) Photomicrograph and (b)

1645 ACF of an Mg-Zr alloy that was initially formed by rolling, which produced the

1646 vertical laminar structure. The horizontal light gray zones are overgrowths that

1647 formed under high temperature conditions. During this deformation, the fabric was

1648 also broken into blocky domains by shear fractures. See Table 1 for results.

1649

1650 Figure 16. Segmentation of the "grains" in the Mg-Zr alloy dramatically changes the

1651 best-fit strain results. (a) The "grains" are manually segmented and assigned

1652 uniform gray scale values. (b) The ACF suggests the segmented grains are elongated

1653 in the vertical direction. Black line marks the $r_{0}^{*}$ contour. See Table 3 for results.

1654

1655 Table 1: Best-fit results for phantom, ooid, and $\mathrm{Mg}-\mathrm{Zr}$ alloy samples.

1656 Table 2: Best-fit results for pressure-solved sandstone sample. 
Click here to download high resolution image

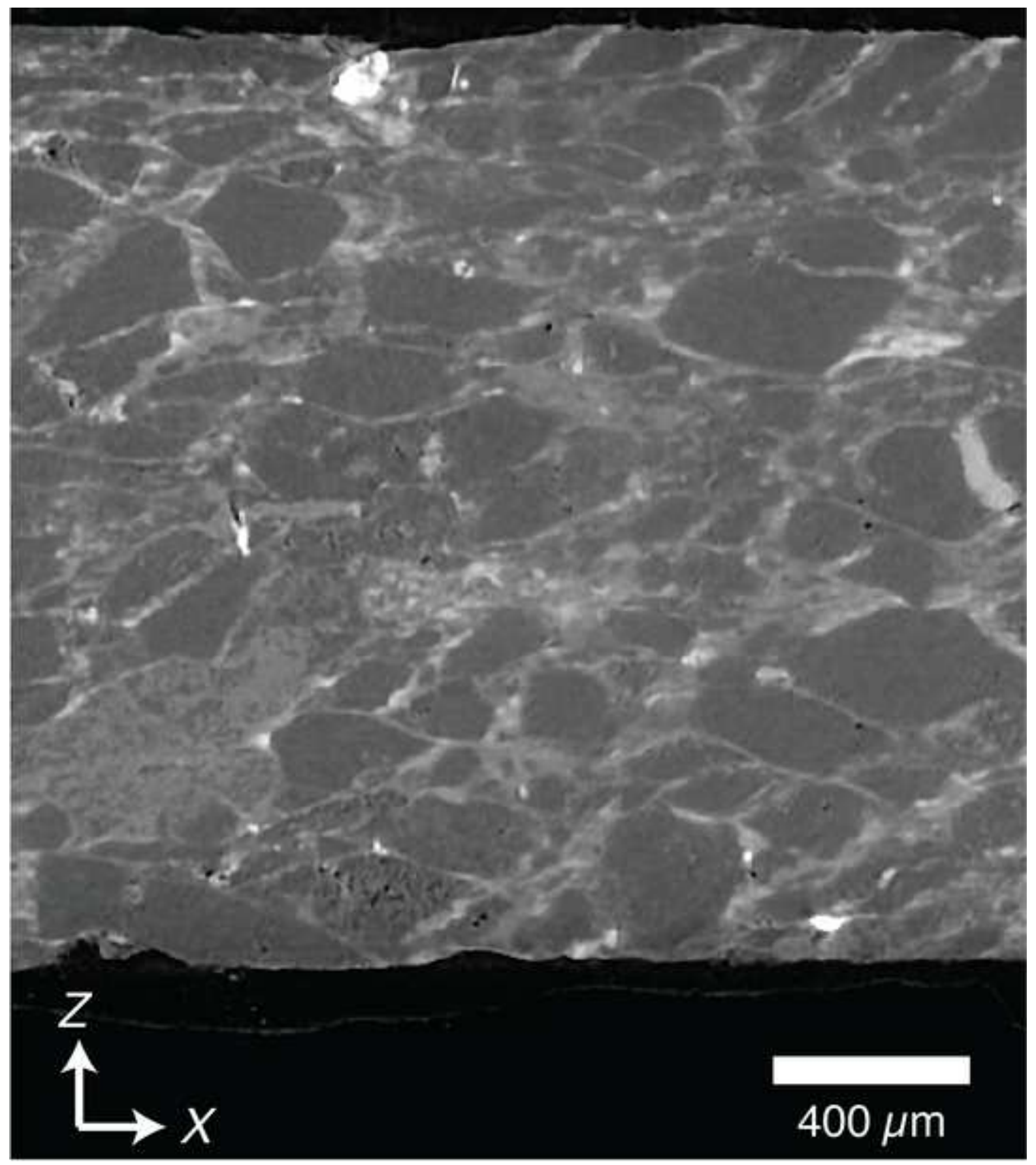




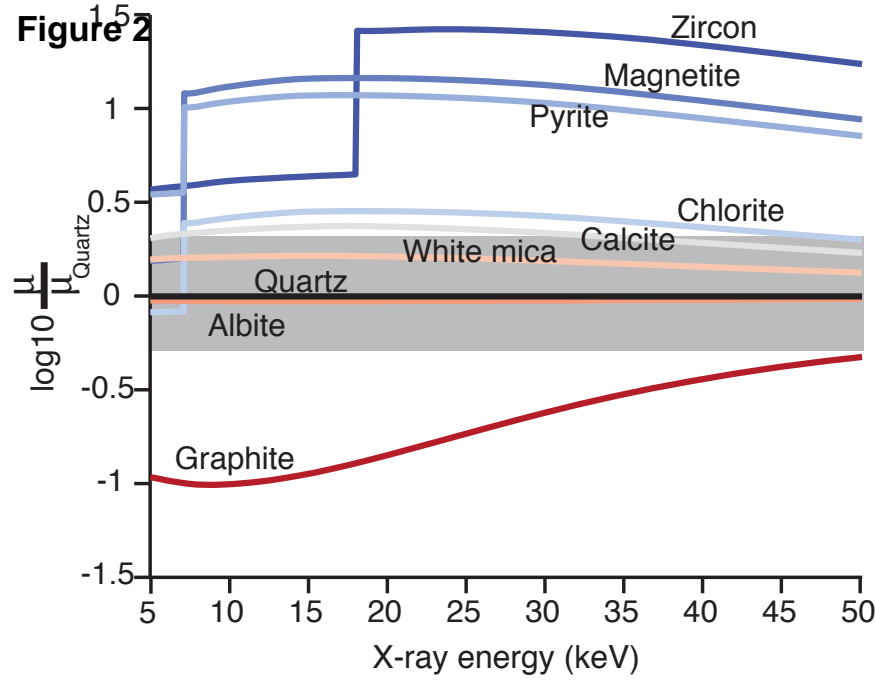


a)Eionre 3

$400 \mu \mathrm{m}$ 
ejguserftgle Circle

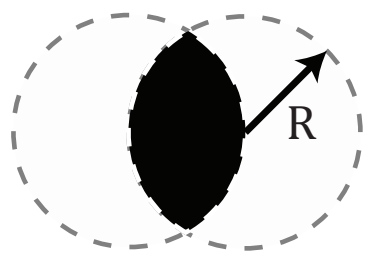

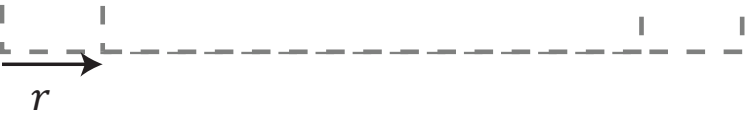

c) Anticlustered

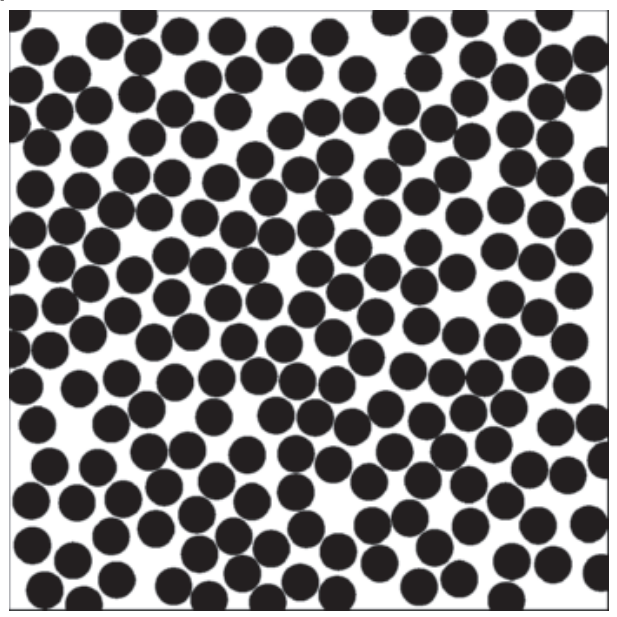

b) Square Lattice

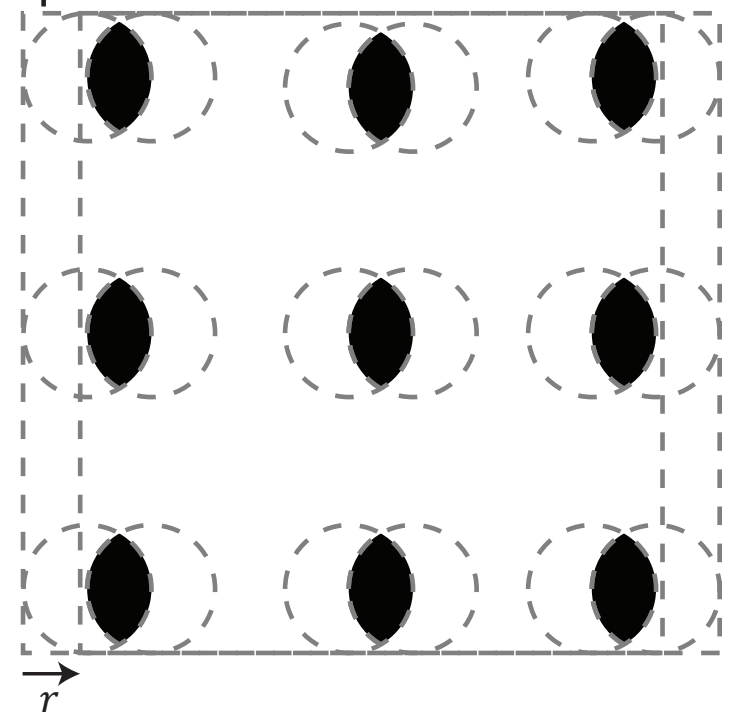

d) Autocorrelation Functions

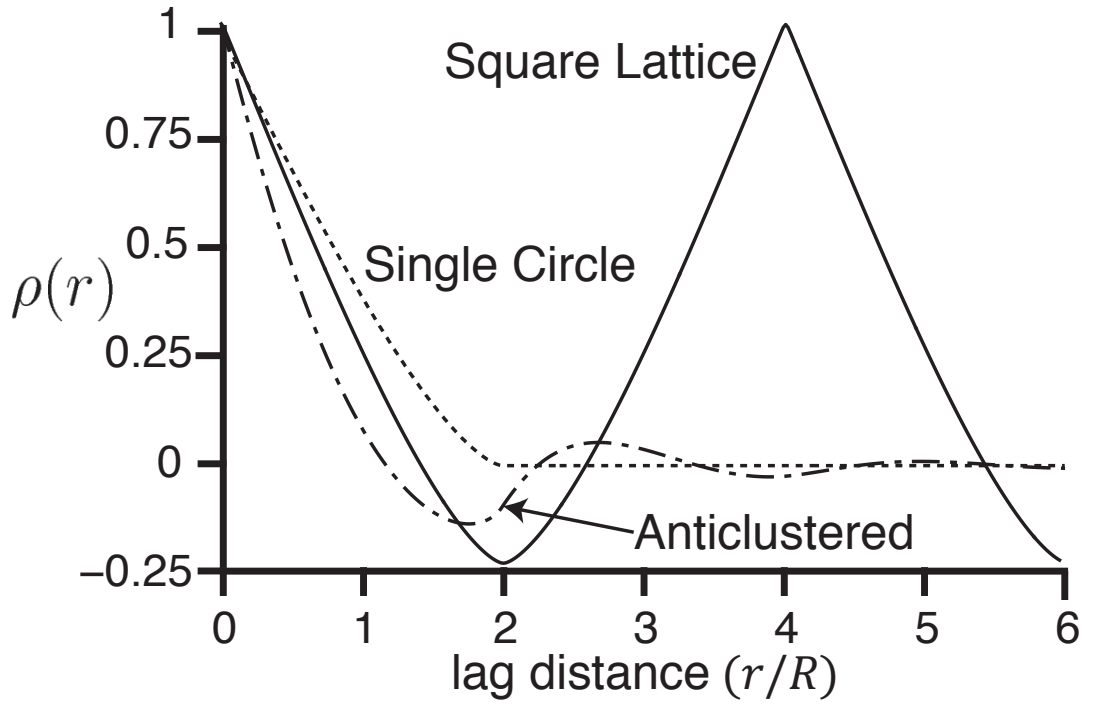


Figure 5

a)

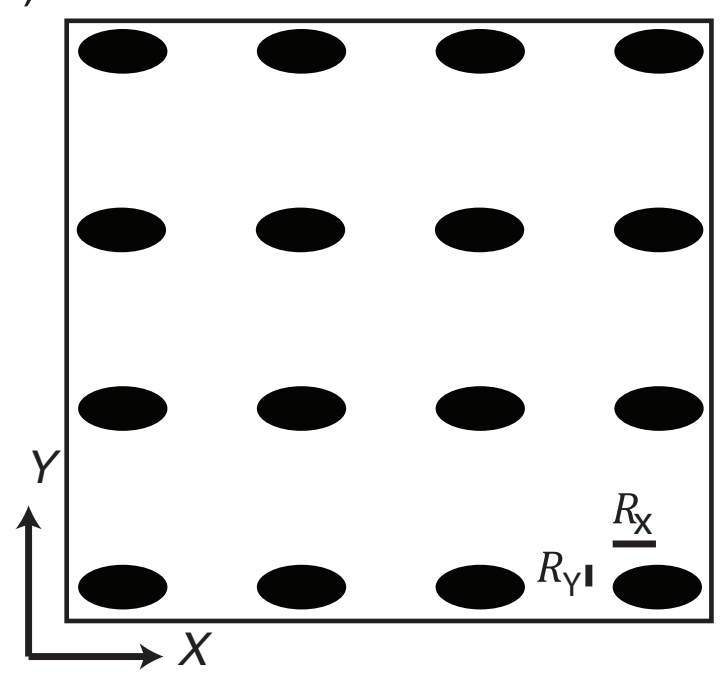

Ramsay Effect

b)

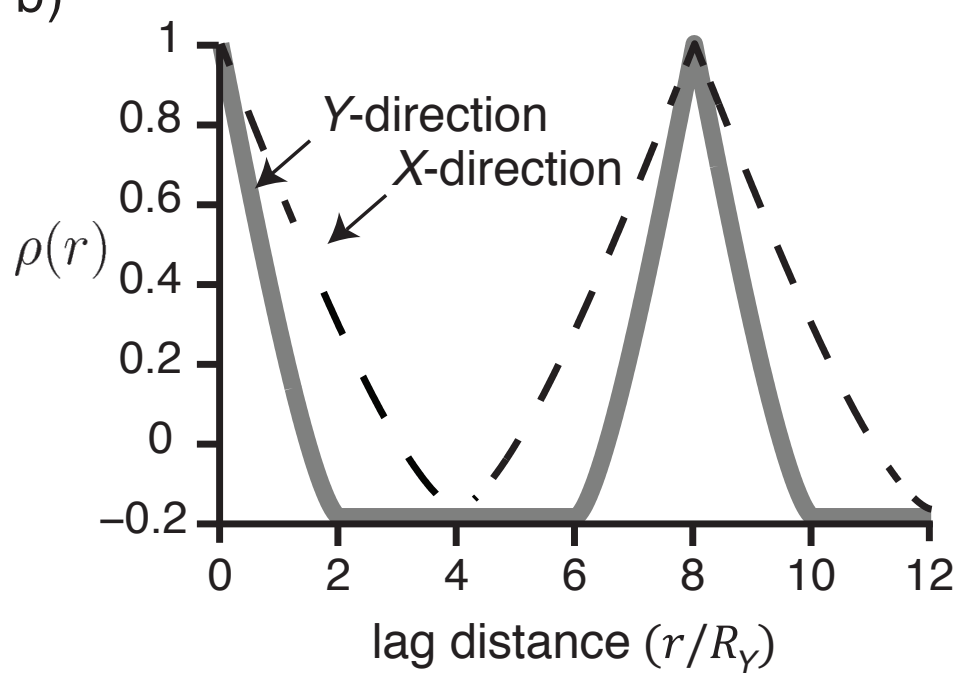

Fry Effect

c)

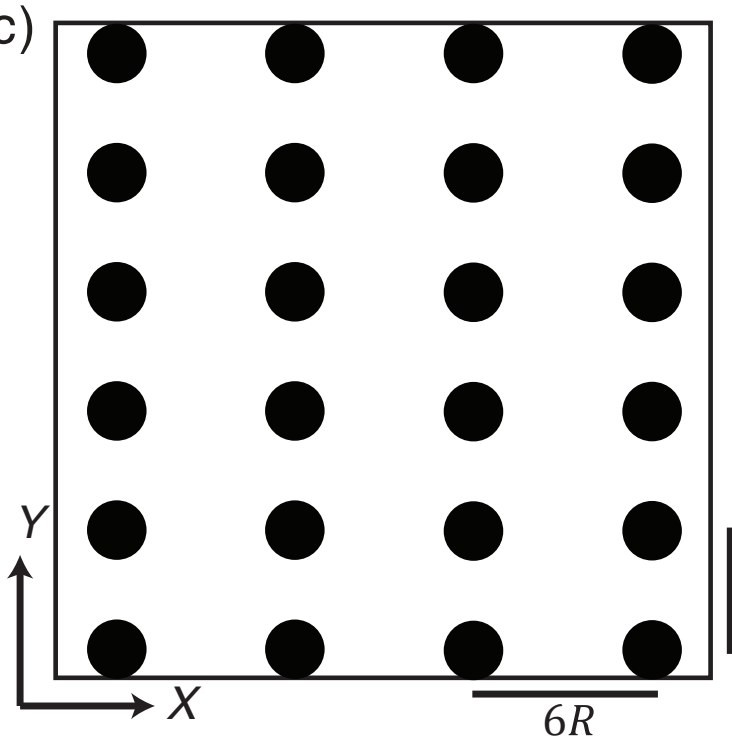

d)

$4 R$
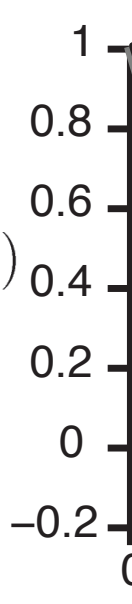

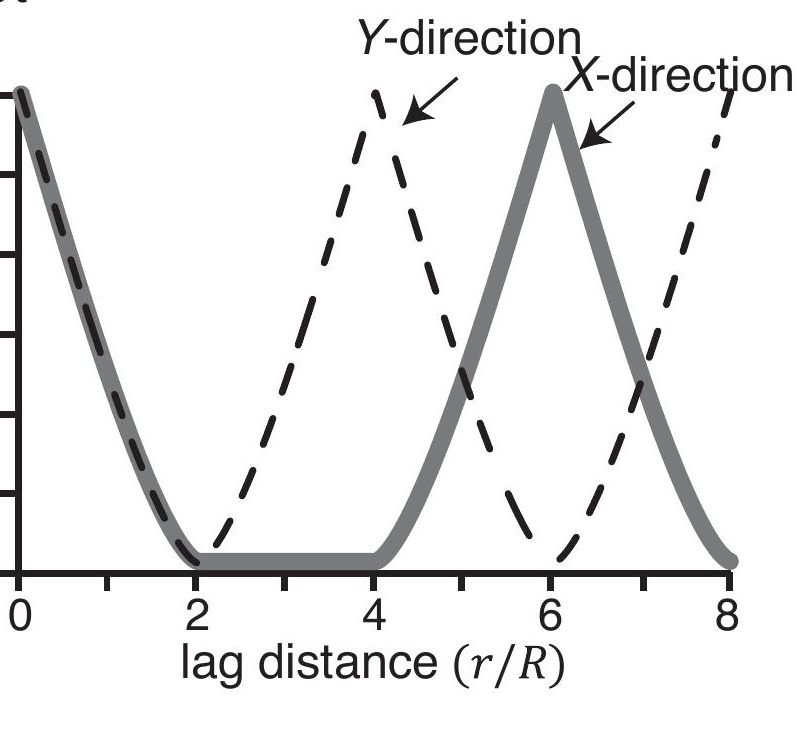




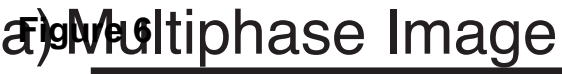

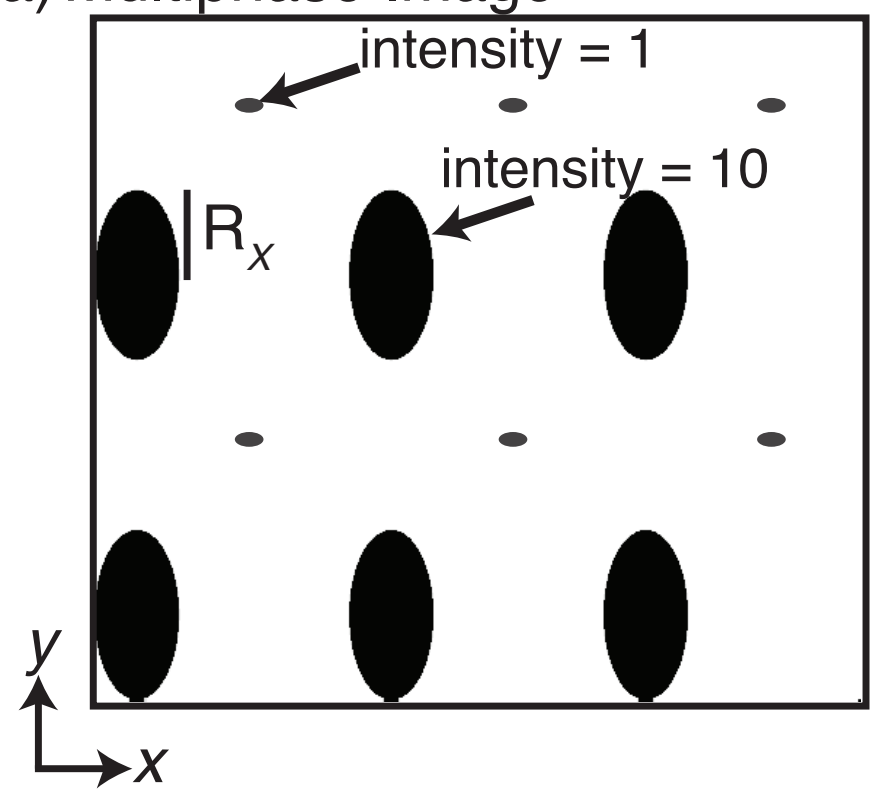

c) Multiphase Image

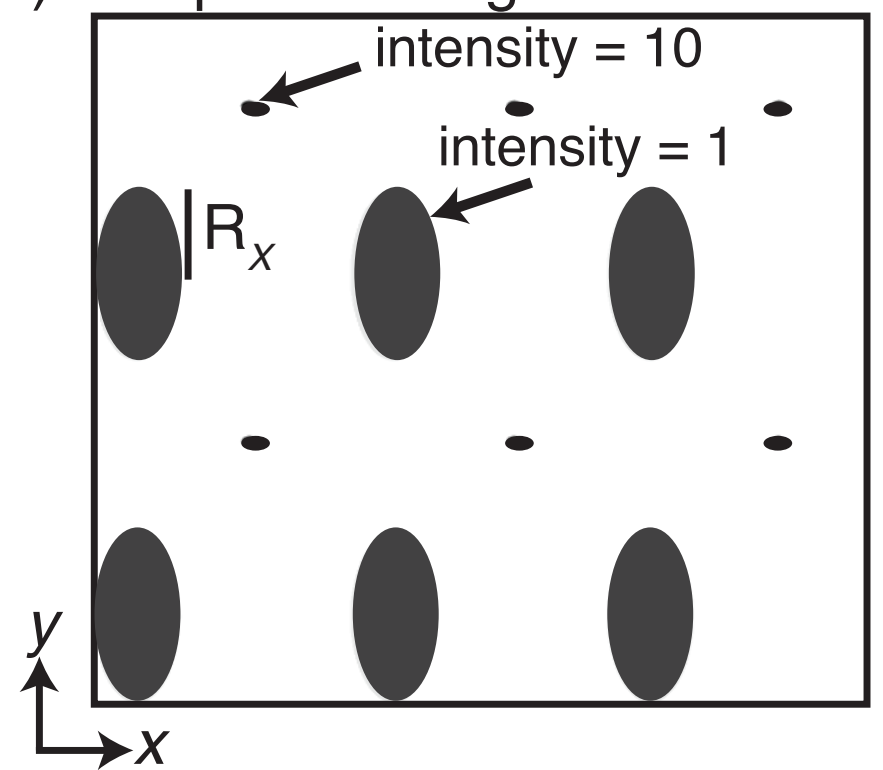

b) $\mathrm{ACF}$

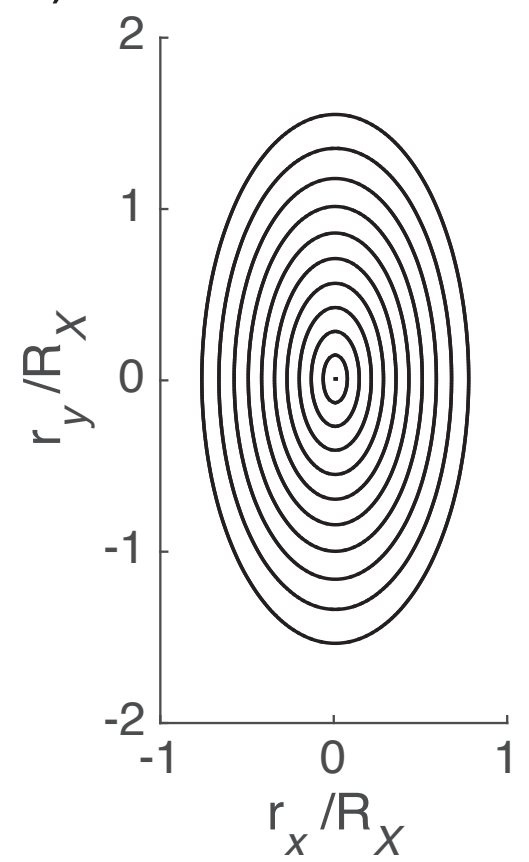

d) $\mathrm{ACF}$

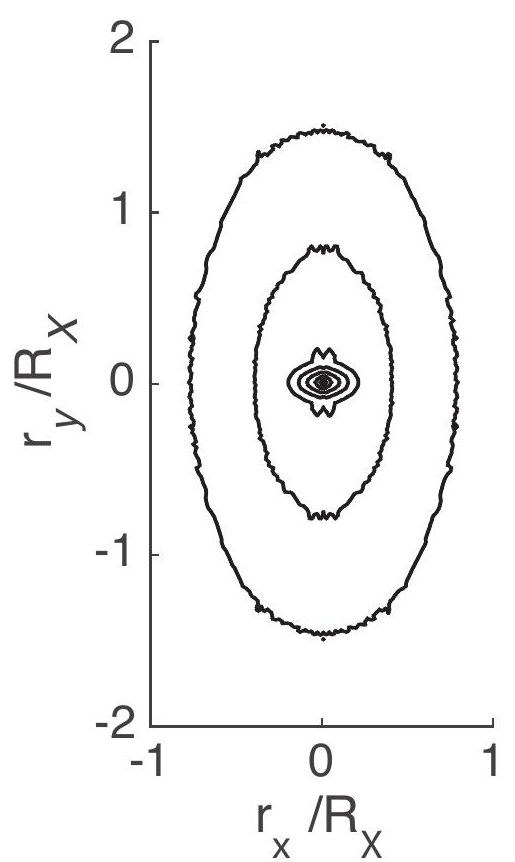


A)igure 7

b)
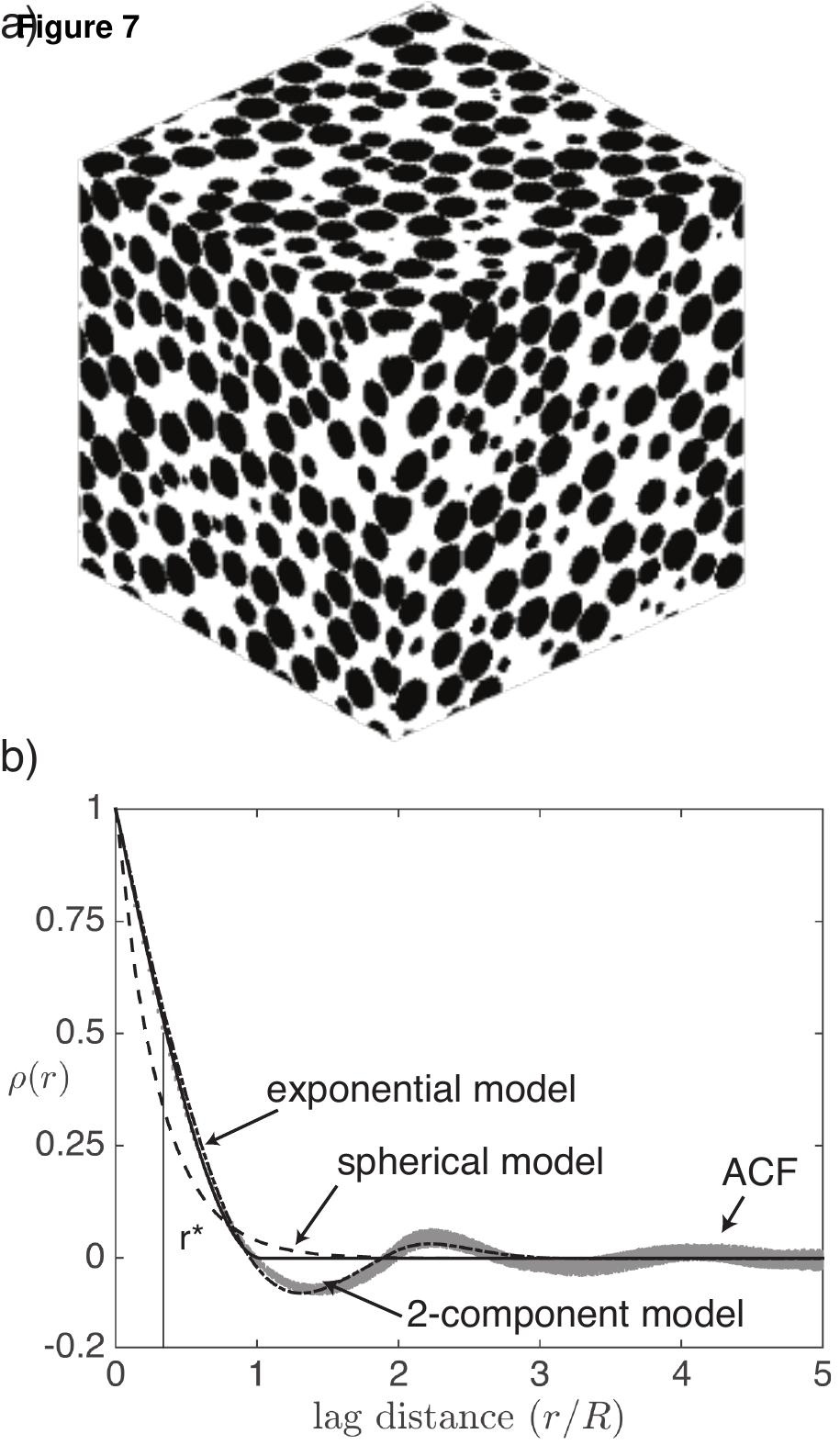

c)

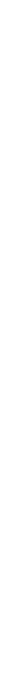




\section{Figure 8}

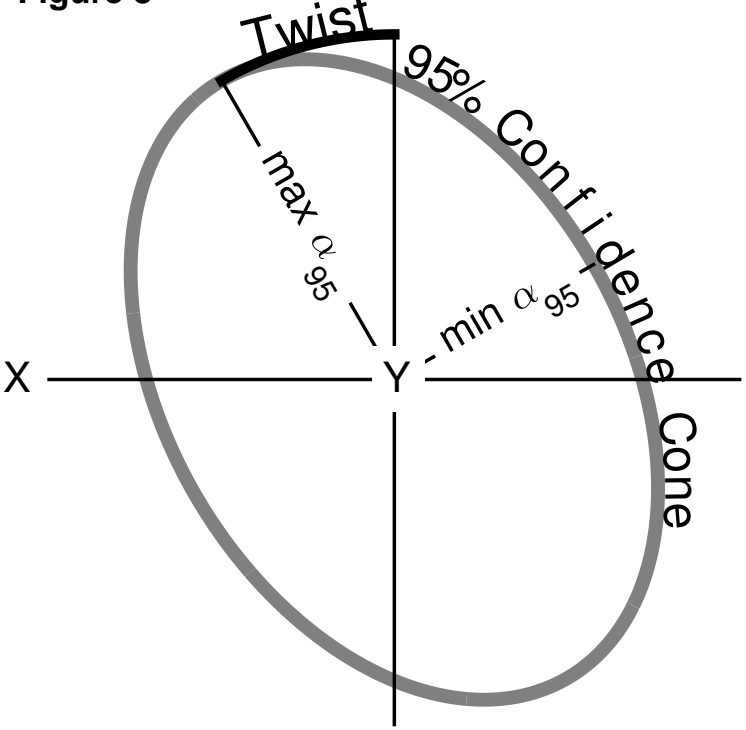


Figure 9 ) Phantom Aggregrate
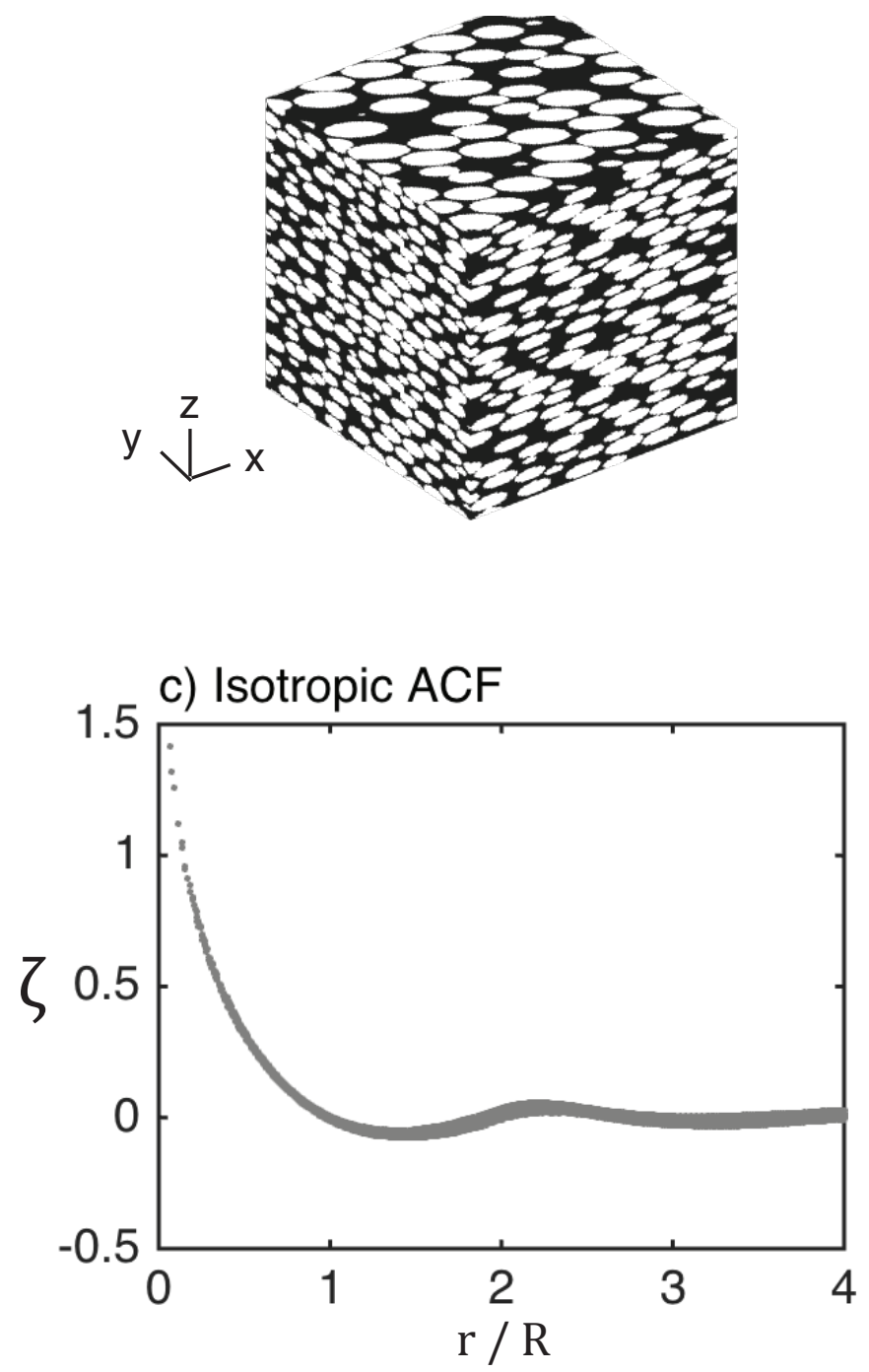
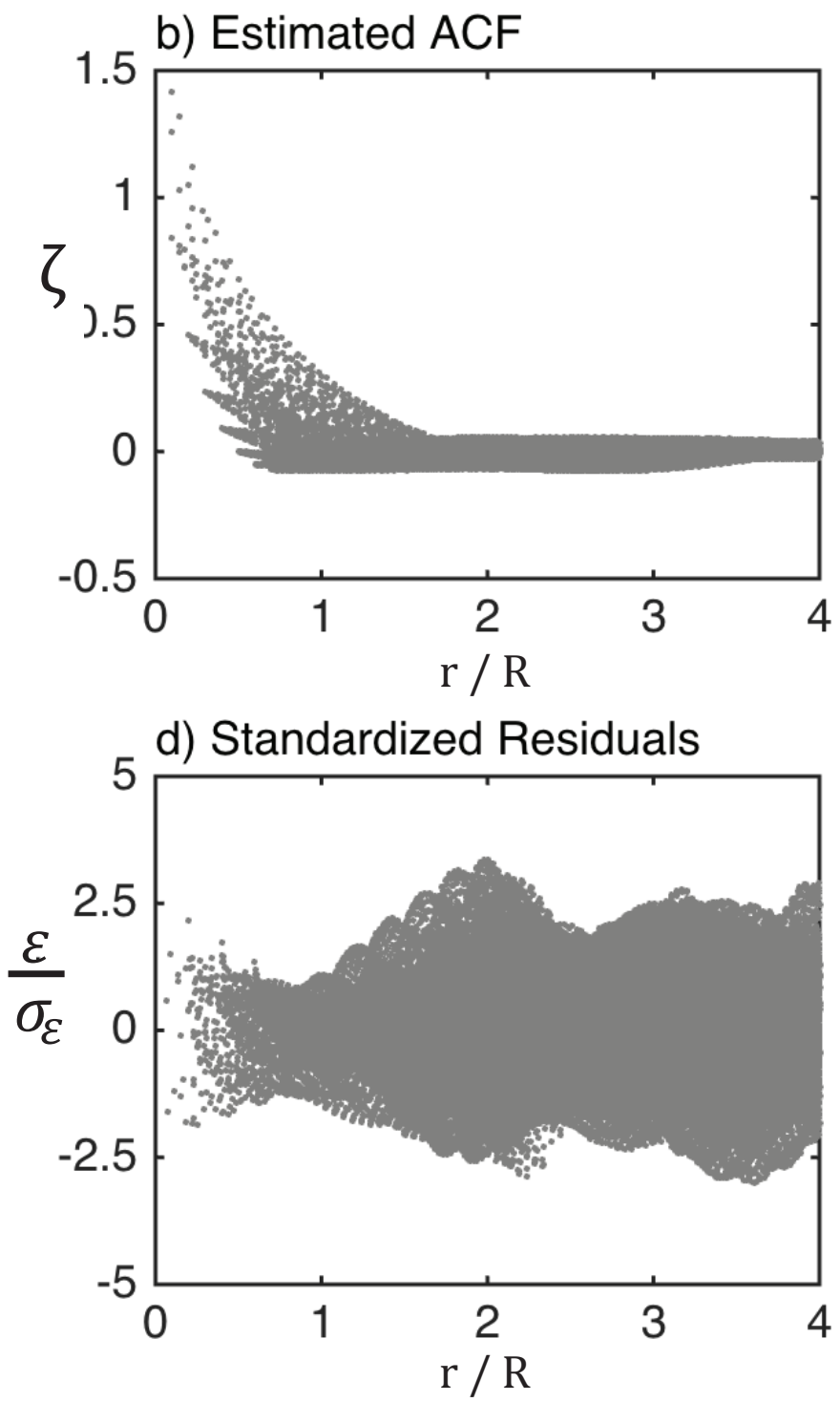
Figure a) Deformed Ooids

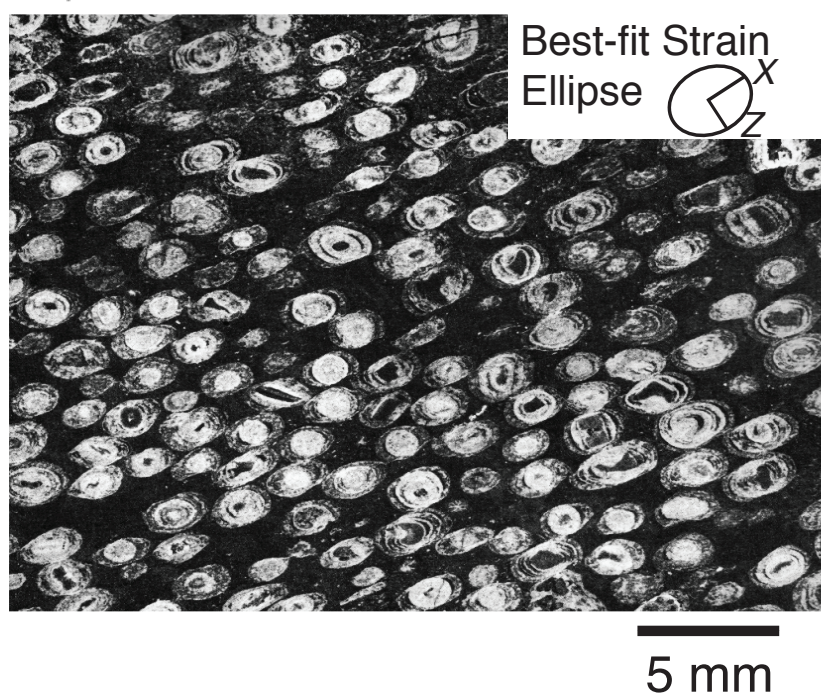

c) Isotropic ACF

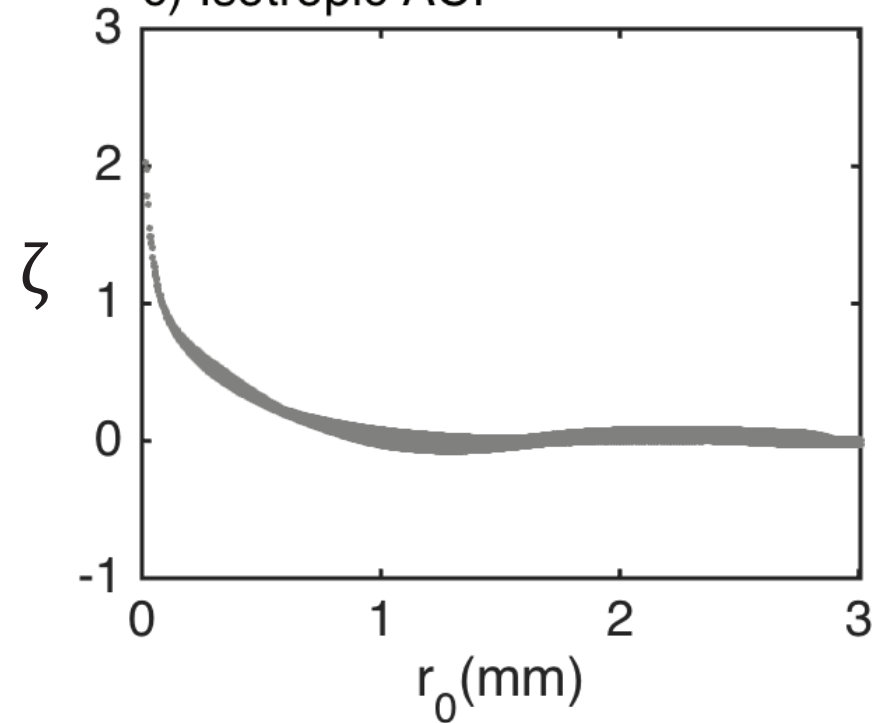

b) Estimated ACF

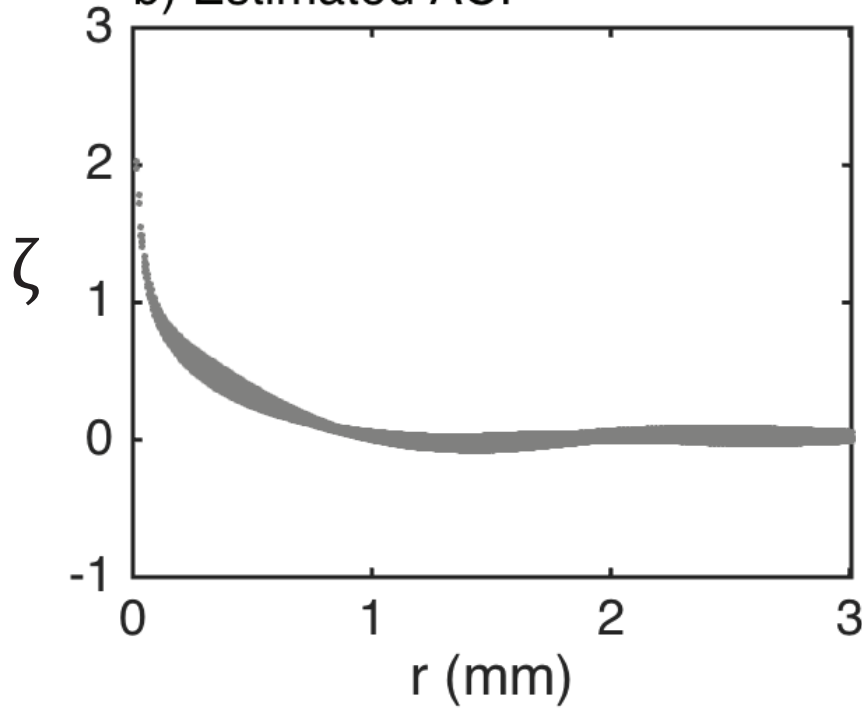

d) Standardized Residuals

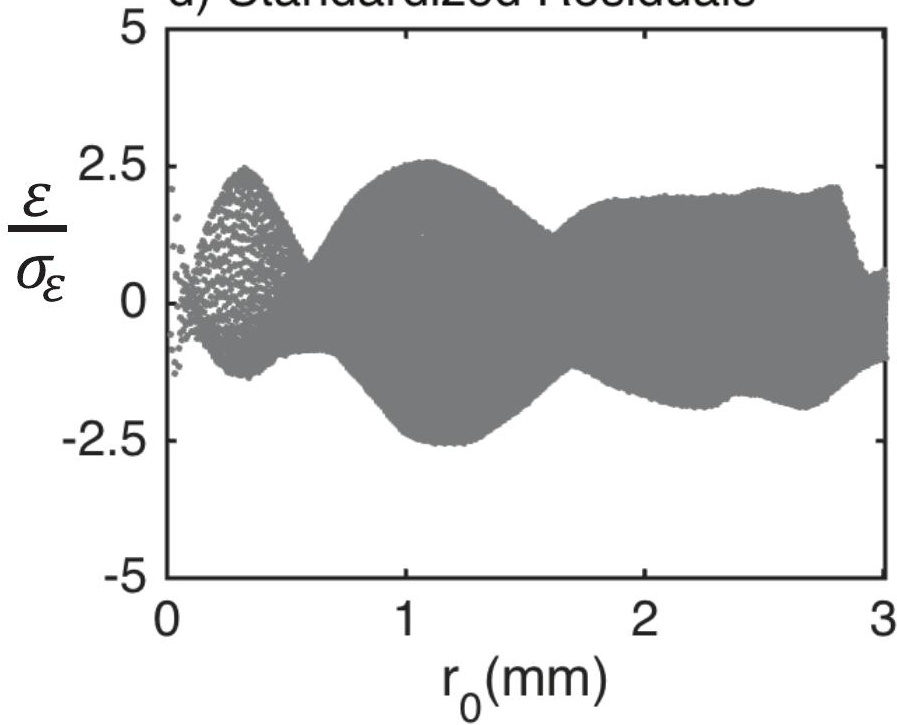




\section{Filitirä 4 grain}

selvage

fibrous

quartz / feldspar

overgrowth

grain 


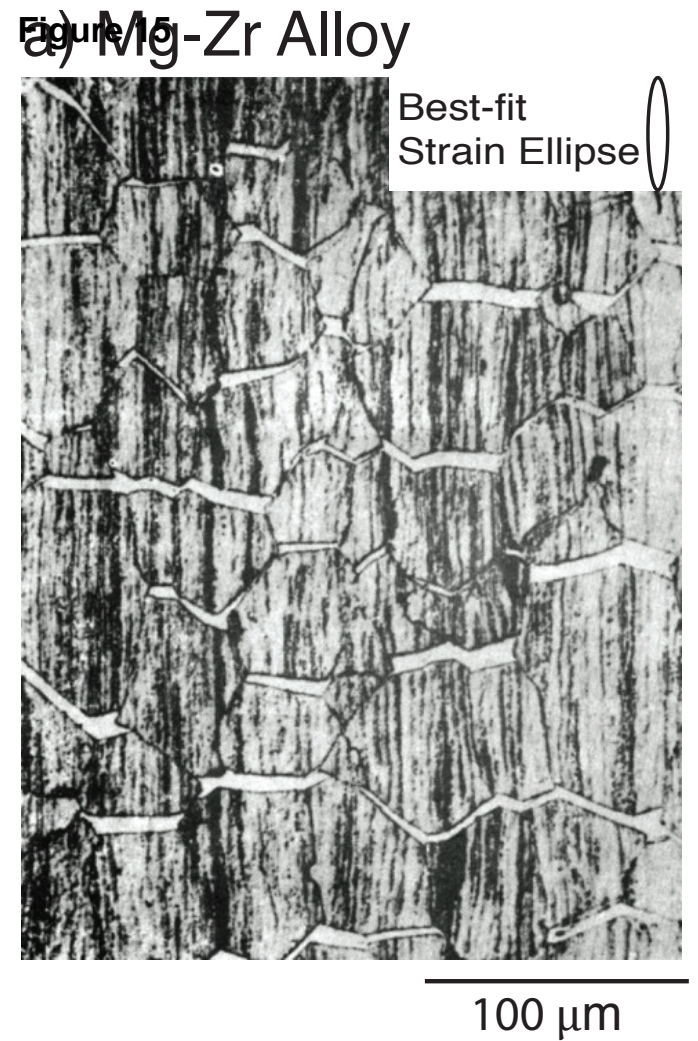

b) $\mathrm{ACF}$

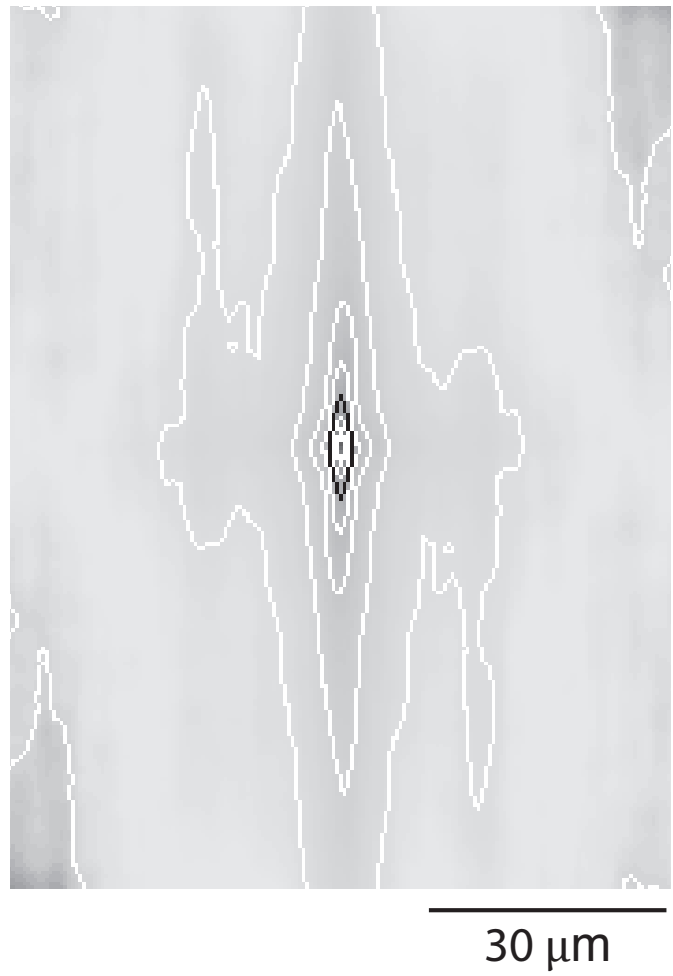



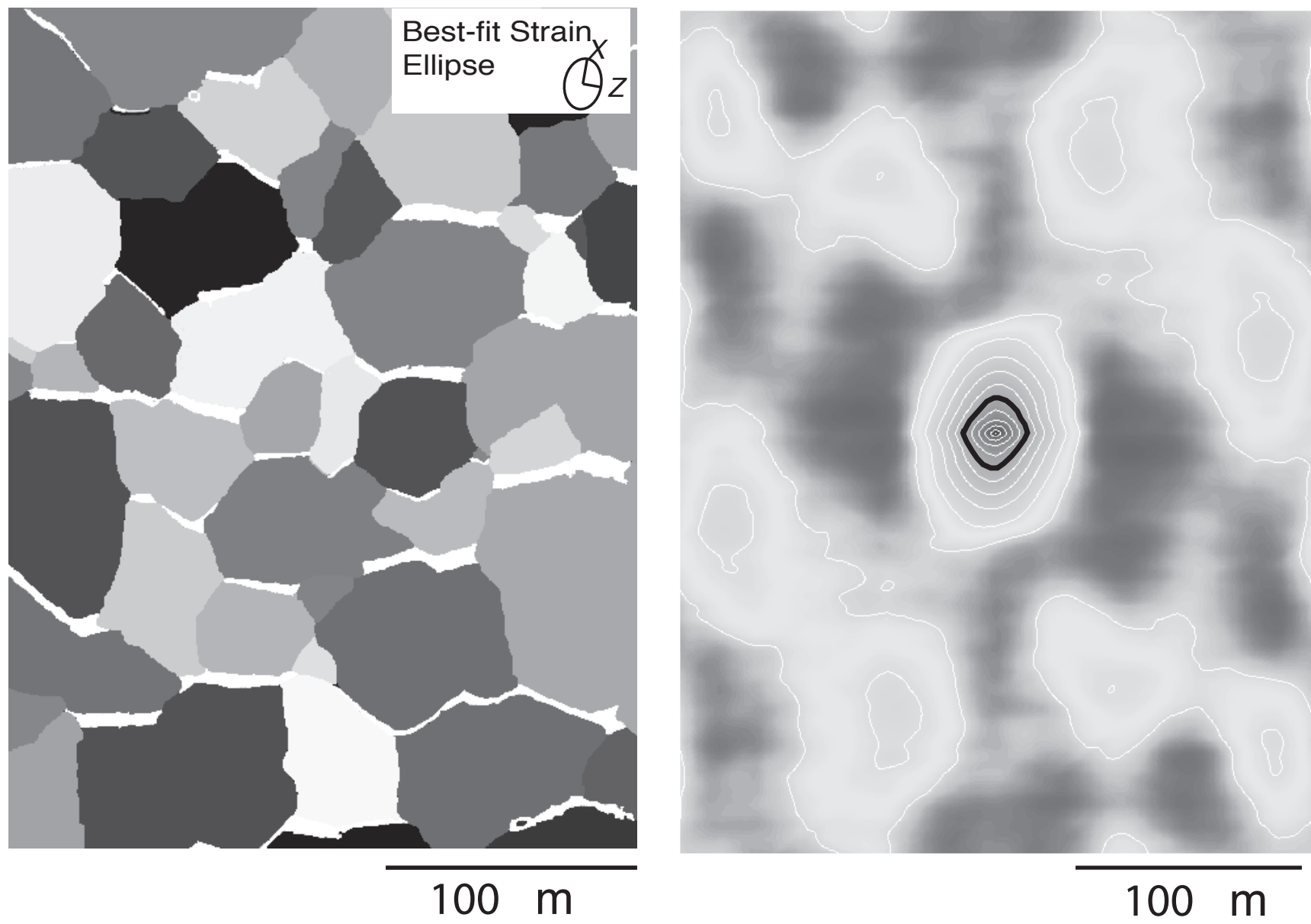
Table 1: Best-fit results for phantom, ooid, and $\mathrm{Mg}-\mathrm{Zr}$ alloy samples.

\begin{tabular}{|c|c|c|c|c|c|c|c|c|c|c|c|}
\hline \multirow[b]{2}{*}{ Sample, Method } & \multicolumn{7}{|c|}{ Principal Magnitudes and Directions } & \multirow{2}{*}{$\begin{array}{c}\text { Isotropy } \\
\text { Prob. } \\
(\%)\end{array}$} & \multirow{2}{*}{$\begin{array}{c}\text { Grain } \\
\text { Radius } \\
R_{\mathrm{S}}(\mu \mathrm{m})\end{array}$} & \multicolumn{2}{|c|}{ Fit } \\
\hline & \multicolumn{2}{|c|}{$\begin{array}{l}\text { Princ. } S^{\prime} \\
\text { Axis } l / l_{0}\end{array}$} & $\begin{array}{c}E^{\prime} \\
(\mathrm{cNp})\end{array}$ & $\begin{array}{l}\operatorname{Tr} / \mathrm{Pl} \\
\text { (deg) }\end{array}$ & $\begin{array}{c}\max \alpha_{95} \\
(\operatorname{deg})\end{array}$ & $\min _{(\mathrm{deg})} \alpha_{95}$ & $\begin{array}{l}\text { Twist } \\
\text { (deg) }\end{array}$ & & & $R^{2}$ & $d$ \\
\hline \multirow{4}{*}{$\begin{array}{l}\text { Phantom } \\
\text { Fig. 9 } \\
\qquad \text { ACF }\end{array}$} & & & & & & & & & & & \\
\hline & $X$ & $2.00(0)$ & $+69.21(5)$ & $330 / 00$ & 0.0 & 0.0 & 01 & Y-Z: 0 & & & \\
\hline & $Y$ & $1.00(0)$ & $-00.36(4)$ & $060 / 00$ & 0.0 & 0.0 & 03 & $\mathrm{X}-\mathrm{Z}: 0$ & $97^{*}$ & 0.96 & 1.97 \\
\hline & $Z$ & $0.50(0)$ & $-68.85(4)$ & $234 / 90$ & 0.0 & 0.0 & 90 & $X-Y: 0$ & & & \\
\hline \multicolumn{12}{|c|}{ Undeformed Oolite } \\
\hline \multirow[t]{4}{*}{ Fig. 10} & $X$ & $1.02(0)$ & $+02.12(8)$ & $121 / 00$ & 0.9 & 0.0 & 90 & Y-Z: 0 & \multirow{2}{*}{160} & \multirow[b]{2}{*}{0.93} & \multirow{2}{*}{1.99} \\
\hline & $Z$ & $0.98(0)$ & $-02.12(8)$ & $031 / 00$ & 0.9 & 0.0 & 00 & $\mathrm{X}-\mathrm{Y}: 0$ & & & \\
\hline & $X$ & $1.00(2)$ & $+1(1)$ & 098/00 & 21 & 57 & \multirow[b]{2}{*}{ - } & \multirow[b]{2}{*}{ - } & \multirow[b]{2}{*}{-} & \multirow{2}{*}{ - } & \multirow{2}{*}{ - } \\
\hline & $Z$ & $1.00(2)$ & $-1(1)$ & $008 / 00$ & 21 & 77 & & & & & \\
\hline \multicolumn{12}{|l|}{ Deformed Oolite } \\
\hline Fig. $11 \quad A C F$ & $X$ & $1.13(0)$ & $+12.2(1)$ & $020 / 00$ & 0.3 & 0.0 & 90 & Y-Z: 0 & \multirow{2}{*}{828} & \multirow{2}{*}{0.94} & \multirow{2}{*}{1.97} \\
\hline & $Z$ & $0.89(0)$ & $-12.2(1)$ & $110 / 00$ & 0.3 & 0.0 & 00 & $X-Y: 0$ & & & \\
\hline$R_{f} / \phi$ & $X$ & $1.09(3)$ & $+8(1)$ & $020 / 00$ & 3 & 3 & - & - & - & - & - \\
\hline inside grain & $Z$ & $0.92(3)$ & $-8(1)$ & $110 / 00$ & 3 & 3 & - & - & - & - & - \\
\hline \multirow{2}{*}{ Nearest Neighbor } & $X$ & 1.34 & +29.11 & $025 / 00$ & - & - & - & - & - & - & 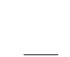 \\
\hline & $Z$ & 0.75 & -29.11 & $115 / 00$ & & & & & - & - & \\
\hline \multicolumn{12}{|l|}{ Mg-Zr Alloy } \\
\hline \multirow{2}{*}{ Fig. $15 \quad \mathrm{ACF}$} & $X$ & $2.41(0)$ & $+87.8(5)$ & $090 / 00$ & 0.1 & 0.0 & 90 & Y-Z: 0 & \multirow{2}{*}{21} & \multirow{2}{*}{0.94} & \multirow{2}{*}{1.41} \\
\hline & $Z$ & $0.42(0)$ & $-87.8(5)$ & $180 / 00$ & 0.1 & 0.0 & 00 & $\mathrm{X}-\mathrm{Y}: 0$ & & & \\
\hline \multicolumn{12}{|c|}{ Mg-Zr Alloy, segmented } \\
\hline \multirow{2}{*}{ Fig. 16 ACF } & $X$ & $1.18(0)$ & $+16.1(1)$ & $080 / 00$ & 0.2 & 0.0 & 90 & Y-Z: 0 & 20 & 0.99 & 1.84 \\
\hline & $Z$ & $0.85(0)$ & $-16.1(1)$ & $170 / 00$ & 0.2 & 0.0 & 00 & $X-Y: 0$ & 20 & 0.99 & 1.84 \\
\hline
\end{tabular}

Errors are reported using concise notation. For example $1.4(3)=1.4 \pm 0.3$

Orientations are reported in sample coordinates.

${ }^{*}$ Grain radius for the Phantom example was scaled to 100 microns for this calculation. 
Table 2: Best-fit results for pressure-solved sandstone sample.

\begin{tabular}{|c|c|c|c|c|c|c|c|c|c|c|}
\hline \multirow[b]{2}{*}{ Sample, Method } & \multicolumn{6}{|c|}{ Principal Magnitudes and Directions } & \multirow{2}{*}{$\begin{array}{c}\text { Isotropy } \\
\text { Prob. } \\
(\%)\end{array}$} & \multirow{2}{*}{$\begin{array}{c}\text { Grain } \\
\text { Radius } \\
R_{\mathrm{S}}(\mu \mathrm{m})\end{array}$} & \multicolumn{2}{|c|}{ Fit } \\
\hline & $\begin{array}{l}\text { Princ. } S_{g} \\
\text { Axis } l / l_{0}\end{array}$ & $\begin{array}{c}E_{g} \\
(\mathrm{cNp})\end{array}$ & $\begin{array}{l}\mathrm{Tr} / \mathrm{Pl} \\
\text { (deg) }\end{array}$ & $\begin{array}{l}\max \alpha_{95} \\
\quad(\operatorname{deg})\end{array}$ & $\begin{array}{c}\min \alpha_{95} \\
(\operatorname{deg})\end{array}$ & $\begin{array}{l}\text { Twist } \\
\text { (deg) }\end{array}$ & & & $R^{2}$ & $d$ \\
\hline \multicolumn{11}{|l|}{$92810-3$} \\
\hline \multirow[t]{4}{*}{ Fig. 13} & $X \quad 1.00(0)$ & $+00.00(5)$ & $263 / 09$ & 0.8 & 0.0 & 00 & Y-Z: 0 & \multirow{4}{*}{149} & \multirow{4}{*}{0.97} & \multirow{4}{*}{1.97} \\
\hline & $Y \quad 0.97(0)$ & $-02.68(5)$ & $169 / 20$ & 0.8 & 0.0 & 00 & $\mathrm{X}-\mathrm{Z}: 0$ & & & \\
\hline & $0.52(0)$ & $-64.91(5)$ & $016 / 68$ & 0.0 & 0.0 & 84 & $\mathrm{X}-\mathrm{Y}: 0$ & & & \\
\hline & $V \quad 0.51(0)$ & $-67.59(9)$ & - & - & - & - & - & & & \\
\hline \multirow{4}{*}{ PDS } & $X \quad 1.00(0)$ & $+00(0)$ & $263 / 09$ & 14 & 14 & \multirow{4}{*}{ - } & \multirow{4}{*}{ 一 } & \multirow{4}{*}{-} & \multirow{4}{*}{-} & \multirow{4}{*}{-} \\
\hline & $Y \quad 1.00(10)$ & $+00(10)$ & $169 / 20$ & 14 & 14 & & & & & \\
\hline & $Z \quad 0.76(8)$ & $-27(10)$ & $016 / 68$ & 14 & 14 & & & & & \\
\hline & $V \quad 0.76(10)$ & $-27(13)$ & - & - & - & & & & & \\
\hline
\end{tabular}

$S_{g X}$ is set to 1 based on observations of overgrowths in the maximum stretch direction.

Errors are reported using concise notation. For example $1.4(3)=1.4 \pm 0.3$

Orientations are reported in geographic coordinates.

PDS measurements and errors are calculated according to Feehan and Brandon (1999). 3D correction introduced by Rahl et al. (2011). 